\title{
CONVERGENCE OF THE SCHRÖDINGER-POISSON SYSTEM TO THE EULER EQUATIONS UNDER THE INFLUENCE OF A LARGE MAGNETIC FIELD
}

\author{
MarJolaine Puel ${ }^{1}$
}

\begin{abstract}
In this paper, we prove the convergence of the current defined from the SchrödingerPoisson system with the presence of a strong magnetic field toward a dissipative solution of the Euler equations.
\end{abstract}

Mathematics Subject Classification. 76X05, 76N99, 81Q99, 82D10, 35Q40.

Received: October 26, 2001. Revised: June 18, 2002.

\section{INTRODUCTION}

We consider in this paper the behavior of electrons moving on a positive charged background influenced by a very strong magnetic field. We describe this phenomenon from a quantum point of view. This model describes a magneto-active plasma where the velocities of the particles are small compared to the speed of light (to use the electrostatic approximation) and where the magnetic field is very strong (like in the magneto-sphere). The equations satisfied by the wave functions are a variation from the Schrödinger-Poisson system. They depend on three parameters, the Planck constant $(h>0)$, the permittivity of the system $(\varepsilon>0)$ and the strength of the magnetic field.

We deal with the asymptotic limit which corresponds to the case when the magnetic field is very strong, the Planck constant and the permittivity are both very small (implying that the electron density is quasi-equal to the ion density). As Brenier in [2], who studied the quasi-neutral limit of the Vlasov-Poisson system (with and without external magnetic field), we obtain at the limit that the curl of the potential involved in the system is a dissipative solution of the Euler equations in the sense of P.-L. Lions.

In [11], we also studied the same asymptotic limit in the case of the Schrödinger-Poisson system without magnetic field and obtained that the electron current converges to a dissipative solution to the Euler equations. Those results are a rigorous justification of the composition of two different asymptotic processes which already have been independently studied in $[8,9]$ and $[5]$ for the semi-classical limit and, as mentioned above, in [2] for the quasi-neutral limit.

Keywords and phrases. Quasi-neutral plasmas, semi-classical limit, modulated energy.

1 Laboratoire d'analyse numérique (B 187), Université Pierre et Marie Curie, 4 Place Jussieu, 75252 Paris Cedex 05, France. e-mail: mpuel@ann.jussieu.fr 


\subsection{Presentation of the system}

We write the Schrödinger equation describing the motion of electrons on a charged background submitted to an external magnetic field $B=\operatorname{curl} A$. In this case ( $c f$. [4]), the quantum Hamiltonian is given by

$$
H_{q}=\frac{1}{2 m}[P-q A]^{2}=\frac{1}{2 m}\left(P^{2}+q A P+q P A+q^{2} A^{2}\right)
$$

where $P$ is the operator defined by $P=\frac{h}{i} \nabla$.

This problem is initially stated in $\mathbb{R}^{3}$. Since we neglect the third component of the velocity and the dependence of the two first ones with respect to the vertical variable, our study is set in $\mathbb{R}^{2}$. In the following, for $Z \in \mathbb{R}^{2}$, we denote by ${ }^{\perp} Z$ the vector

$$
{ }^{{ }} Z=\left(\begin{array}{c}
Z_{2} \\
-Z_{1}
\end{array}\right)
$$

We choose a potential $A=-\frac{\perp_{x}}{2 \varepsilon}$, which corresponds to a vertical magnetic field. Let us notice that $A$ commutes with $P$ because $\nabla\left(\frac{\perp_{x}}{2 \varepsilon}\right)=0$. Assuming $q=m=1$, the Schrödinger equation $i h \partial_{t} \psi=H_{q} \psi$ becomes in our setting

$$
i h \partial_{t} \psi^{\varepsilon}+\frac{h^{2}}{2} \Delta \psi^{\varepsilon}+\frac{i h}{2 \varepsilon}\left({ }^{\perp} x \cdot \nabla\right) \psi^{\varepsilon}-\frac{|x|^{2}}{8 \varepsilon^{2}} \psi^{\varepsilon}=0
$$

where $\psi$ is a wave function.

In this paper, we consider a superposition of states (instead of a pure state) where each state $k \in \mathbb{Z}^{2}$ has a weight $\lambda_{k} \geq 0$ and we add the effect of the electric field. Finally, we deal with an electron density given by

$$
\rho^{\varepsilon}(t, x)=\sum_{k \in \mathbb{Z}^{2}} \lambda_{k} \psi_{k}^{\varepsilon}(t, x) \bar{\psi}_{k}^{\varepsilon}(t, x)
$$

where the wave functions satisfy the equation

$$
i h \partial_{t} \psi_{k}^{\varepsilon}+\frac{h^{2}}{2} \Delta \psi_{k}^{\varepsilon}+\frac{i h}{2 \varepsilon}\left({ }^{\perp} x \cdot \nabla\right) \psi_{k}^{\varepsilon}-\frac{|x|^{2}}{8 \varepsilon^{2}} \psi_{k}^{\varepsilon}=\frac{1}{\varepsilon} \phi^{\varepsilon} \psi_{k}^{\varepsilon}
$$

coupled with the Poisson equation

$$
\rho^{\varepsilon}=\theta^{\varepsilon}-\Delta \phi^{\varepsilon}
$$

Here, the ion density $\theta^{\varepsilon}(x) \in L^{\infty}\left(\mathbb{R}^{d}\right)$ satisfies

$$
\begin{gathered}
(1+|x|) \theta \text { is in } L^{1}\left(\mathbb{R}^{2}\right) \cap L^{2}\left(\mathbb{R}^{2}\right), \\
0 \leq \theta^{\varepsilon}(x) \leq 1, \\
\left.\theta^{\varepsilon}(x)=1 \text { if } x \in\right]-R^{\varepsilon} ; R^{\varepsilon}\left[{ }^{d},\right. \\
\left.\theta^{\varepsilon}(x)=0 \text { if } x \in \mathbb{R}^{d} \backslash\right]-R^{\varepsilon}-\gamma^{\varepsilon} ; R^{\varepsilon}+\gamma^{\varepsilon}\left[{ }^{d},\right.
\end{gathered}
$$


where $\left(R^{\varepsilon}\right) \subset \mathbb{R}_{+}^{*}, \quad\left(R^{\varepsilon}\right) \stackrel{\varepsilon \rightarrow 0}{\longrightarrow}+\infty$, and $\left(\gamma^{\varepsilon}\right) \subset \mathbb{R}_{+}^{*}, \quad\left(\gamma^{\varepsilon}\right) \stackrel{\varepsilon \rightarrow 0}{\longrightarrow} 0$. Under this form, $\left(\theta^{\varepsilon}\right)$ converges in $\mathcal{D}^{\prime}\left(\mathbb{R}^{2}\right)$ to 1 and its derivatives to 0 . This is important because we need at the limit a uniform density $\theta$ so that $\nabla \cdot(\theta v)=0$ when $\nabla \cdot v=0$. Moreover, in the existence proof, we need some integrability properties for $\theta^{\varepsilon}$ which are certainly true if $\theta^{\varepsilon}$ is compactly supported.

An adaptation of some results of [1] and [3] enables us to show the existence of a solution of the system (1.2, 1.3) for a fixed $\varepsilon$. We skip the index $\varepsilon$ to present the existence result.

Let us define the following Hilbert spaces

$$
\begin{gathered}
X=\left\{\Gamma=\left(\gamma_{m}\right)_{m \in \mathbb{Z}^{2}} \mid \gamma_{m} \in L^{2}\left(\mathbb{R}^{2}\right), \forall m \text { and } \sum_{m=1}^{\infty} \lambda_{m} \|\left.\gamma_{m}\right|_{L^{2}\left(\mathbb{R}^{2}\right)} ^{2}<\infty\right\}, \\
H=\left\{\gamma \mid\left(\gamma, \nabla \gamma, \Delta \gamma,|x| \gamma,|x| \nabla \gamma,|x|^{2} \gamma\right) \in L^{2}\left(\mathbb{R}^{2}\right)\right\}
\end{gathered}
$$

with their associated norms

$$
\begin{gathered}
\|\Gamma\|_{X}^{2}=\sum_{m=1}^{\infty} \lambda_{m}\left\|\gamma_{m}\right\|_{L^{2}\left(\mathbb{R}^{2}\right)}^{2}, \\
\|\gamma\|_{H}^{2}=\|\gamma\|_{H^{1}\left(\mathbb{R}^{2}\right)}^{2}+\|\Delta \gamma\|_{L^{2}\left(\mathbb{R}^{2}\right)}^{2}+\left|\left\|x \left|\gamma \left\|_{L^{2}\left(\mathbb{R}^{2}\right)}^{2}+\left|\left\|x \left|\nabla \gamma \left\|_{L^{2}\left(\mathbb{R}^{2}\right)}^{2}+\left|\left\|\left.x\right|^{2} \gamma\right\|_{L^{2}\left(\mathbb{R}^{2}\right)}^{2},\right.\right.\right.\right.\right.\right.\right.\right.\right. \\
Y=\left\{\Gamma=\left(\gamma_{m}\right)_{m \in \mathbb{Z}^{2}} \mid \gamma_{m} \in H, \forall m \text { and } \sum_{m=1}^{\infty} \lambda_{m}\left\|\gamma_{m}\right\|_{H}^{2}<\infty,\right\}
\end{gathered}
$$

with the norm

$$
\|\Gamma\|_{Y}^{2}=\sum_{m=1}^{\infty} \lambda_{m}\left\|\gamma_{m}\right\|_{H}^{2}
$$

and finally, if $\theta: \mathbb{R}^{2} \rightarrow \mathbb{R}$,

$$
\tilde{Y}=\left\{\Gamma \in Y \mid \int_{\mathbb{R}^{2}}(\theta-n(\Gamma)) \mathrm{d} x=0\right\}
$$

where $n(\Gamma)=\sum_{m=1}^{\infty} \lambda_{m}\left|\gamma_{m}\right|^{2}$.

The following existence result holds.

Proposition 1.1. Under the following assumptions,

- the function $\theta$ is in $L^{\infty}\left(\mathbb{R}^{2}\right)$ and $(1+|x|) \theta$ is in $L^{1}\left(\mathbb{R}^{2}\right) \cap L^{2}\left(\mathbb{R}^{2}\right)$,

- the initial data are smooth enough, i.e. $\left(\psi_{0, k}\right)_{k \in \mathbb{Z}^{2}} \in \tilde{Y}$,

there exists a unique solution to (1.2-1.3) $\left(\psi_{k}\right)_{k \in \mathbb{Z}^{2}} \in C([0, \infty), Y) \cap C^{1}([0, \infty), X)$ and $\left(\psi_{k}(t)\right)_{k \in \mathbb{Z}^{2}} \in \tilde{Y}$ for each time $t$.

The proof of Proposition 1.1 is very similar to the proof of the existence result in [1] for the pure electrostatic case. The main differences are the following.

First, the definition of the space $H$ is different because of the shape of the operator. Indeed, we need that $|x| \nabla \gamma$ and $|x|^{2} \gamma$ belong to $L^{2}\left(\mathbb{R}^{2}\right)$. This implies some additional computations which can be found in [11]. 
Moreover, we don't get directly that the conservation of energy gives a time independent bound for $\|\psi\|_{H^{1}\left(\mathbb{R}^{2}\right)}$. However, we obtain using the equation that

$$
\frac{\mathrm{d}}{\mathrm{d} t}\left(|| h \nabla \psi_{k}^{\varepsilon}\left\|_{L^{2}}+|||x| \psi_{k}^{\varepsilon}\right\|_{L^{2}}\right) \leq C|| h \nabla \psi_{k}^{\varepsilon}+i \frac{{ }^{\perp} x}{2 \varepsilon} \psi_{k}^{\varepsilon} \|_{L^{2}} \leq C^{\prime} .
$$

It implies (see [1]) that

$$
\left\|\phi^{\varepsilon}\right\|_{L^{\infty}}+\left\|\nabla \phi^{\varepsilon}\right\|_{L^{\infty}}+\left\|\Delta \phi^{\varepsilon}\right\|_{L^{p}} \leq E_{1}+E_{2} t^{2}, \text { for } 1 \leq p<\infty
$$

with $E_{1}$ and $E_{2}$ two constants depending only on $\theta^{\varepsilon}$ and on the initial energy. That gives us as in [1] the existence result for global solutions.

Remark 1.1. It is probably possible to obtain additional regularity for the solution $\psi$ when the initial data are smooth, but this question is not addressed in this work.

We will use this result for any $\varepsilon$. Let us note that since the ion densities $\theta^{\varepsilon}$ are in $L^{\infty}\left(\mathbb{R}^{2}\right)$ and are compactly supported, the first assumption is satisfied.

\subsection{Formal analysis}

In this section, we use the Wigner transform to motivate formally the obtained result. Let us introduce

$$
W^{\varepsilon, h}(t, x, \xi)=(2 \pi h)^{-d} \int_{\mathbb{R}^{d}} \sum_{k} \lambda_{k} \mathrm{e}^{-i \frac{\xi}{h} \cdot z} \psi_{k}^{\varepsilon}\left(t, x+\frac{z}{2}\right) \bar{\psi}_{k}^{\varepsilon}\left(t, x-\frac{z}{2}\right) \mathrm{d} z,
$$

the Wigner transform for the system and let us operate a change of variable

$$
\tilde{W}^{\varepsilon}(t, x ; \tilde{\xi})=W^{\varepsilon}(t, x, \xi) \text { with } \tilde{\xi}=\xi+\frac{{ }^{\perp} x}{2 \varepsilon} .
$$

The new function $\tilde{W}^{\varepsilon}(t, x ; \tilde{\xi})$ satisfies

$$
\partial_{t} \tilde{W}^{\varepsilon}+\tilde{\xi} \cdot \nabla_{x} \tilde{W}^{\varepsilon}+\frac{1}{\varepsilon} \perp \tilde{\xi} \cdot \nabla_{\tilde{\xi}} \tilde{W}^{\varepsilon}+\frac{1}{\varepsilon} \tilde{K} * \tilde{W}^{\varepsilon}=0
$$

and

$$
\Delta \phi^{\varepsilon}=\rho^{\varepsilon}-\theta^{\varepsilon}
$$

with

$$
\tilde{K}(t, x, \tilde{\xi})=\frac{i}{(2 \pi)^{d}} \int_{\mathbb{R}^{d}} \mathrm{e}^{-i \tilde{\xi} \cdot y} \frac{\left(\phi^{\varepsilon}\left(t, x+\frac{h y}{2}\right)-\phi^{\varepsilon}\left(t, x-\frac{h y}{2}\right)\right)}{h} \mathrm{~d} y
$$

and

$$
\tilde{\rho}^{\varepsilon}(t, x)=\int \tilde{W}^{\varepsilon}(t, x, \tilde{\xi}) \mathrm{d} \tilde{\xi}
$$

Denoting $\tilde{J}^{\varepsilon}(t, x)$ the new current defined by

$$
\tilde{J}_{i}^{\varepsilon}(t, x)=\int \tilde{\xi}_{i} \tilde{W}^{\varepsilon}(t, x, \tilde{\xi}) \mathrm{d} \tilde{\xi},
$$


we obtain the following equations

$$
\begin{gathered}
\partial_{t} \tilde{\rho}^{\varepsilon}+\nabla \cdot \tilde{J}^{\varepsilon}=0 \\
\partial_{t} \tilde{J}_{i}^{\varepsilon}(t, x)=-\int \tilde{\xi}_{i} \tilde{\xi}_{j} \partial_{j} \tilde{W}^{\varepsilon}(t, x, \tilde{\xi}) \mathrm{d} \tilde{\xi}+\frac{1}{\varepsilon}\left({ }^{\perp} \tilde{J}_{i}^{\varepsilon}(t, x)-\partial_{i} \phi^{\varepsilon}(t, x) \tilde{\rho}^{\varepsilon}(t, x)\right) .
\end{gathered}
$$

Therefore, we obtain formally, when $\epsilon$ goes to zero that

$$
\begin{gathered}
\tilde{J}=-\tilde{\rho}^{\perp} \nabla \phi, \\
\partial_{t} \tilde{\rho}+\nabla \cdot \tilde{J}=0
\end{gathered}
$$

and

$$
\tilde{\rho}-1=\Delta \phi .
$$

Those equations are nothing but the incompressible Euler system written for $w=\operatorname{curl} u=\tilde{\rho}-1, u=-{ }^{\perp} \nabla \phi$ since we have

$$
\begin{gathered}
\partial_{t} w+\nabla \cdot(u w)=0, \\
\nabla \cdot u=0 .
\end{gathered}
$$

\subsection{Main result}

Theorem 1.2. Let $T>0$ and $J^{0}=-{ }^{\perp} \nabla \Phi_{0}$ be a divergence free vector in $L^{2}\left(\mathbb{R}^{2}\right)$. For any $\varepsilon>0$, let $\theta^{\varepsilon}$ be the ion density of charge characterized by (1.4-1.6), and let us consider, for any $\varepsilon, h$ and each state $k$, the wave functions $\psi_{k}^{\varepsilon}$, solutions to (1.2, 1.3) with initial data in $\tilde{Y}$. Moreover, we assume that

$$
\begin{gathered}
\int_{\mathbb{R}^{2}} \sum_{k \in \mathbb{Z}^{2}} \lambda_{k}\left|\psi_{k}^{\varepsilon}(0, x)\right|^{2} \mathrm{~d} x=\int_{\mathbb{R}^{2}} \theta^{\varepsilon}(x) \mathrm{d} x, \\
\int_{\mathbb{R}^{2}}\left|\nabla \phi^{\varepsilon}(0, x)-\left({ }^{\perp} J^{0}(x)\right)\right|^{2} \mathrm{~d} x \stackrel{\varepsilon, h \rightarrow 0}{\longrightarrow} 0, \\
\varepsilon \sum_{k \in \mathbb{Z}^{2}} \lambda_{k} \int_{\mathbb{R}^{2}}\left|h \nabla \psi_{k}^{\varepsilon}(0, x)+i \frac{{ }^{\perp} x}{2 \varepsilon} \psi_{k}^{\varepsilon}(0, x)\right|^{2} \stackrel{\varepsilon, h \rightarrow 0}{\longrightarrow} 0 .
\end{gathered}
$$

Then, up to a subsequence, $\left({ }^{\perp} \nabla \phi^{\varepsilon}\right)$ converges in $C^{0}\left([0, T] ; \mathcal{D}^{\prime}\left(\mathbb{R}^{2}\right)\right)$ to a dissipative solution of the Euler equations with $J^{0}$ as initial datum.

Remark 1.3. As in [2], we consider that $J$ is a dissipative solution of the Euler equations with $J^{0}$ as the initial datum if, for all smooth divergence free compactly supported vector $v(t, x)$, and for almost every time,

$$
\begin{aligned}
\int|J(t, x)-v(t, x)|^{2} \mathrm{~d} x \leq & \int\left|J^{0}(x)-v(0, x)\right|^{2} \mathrm{~d} x \exp \left(\int_{0}^{t} 2\|d(v(\theta))\| \mathrm{d} \theta\right) \\
& +2 \int_{0}^{t} \exp \left(\int_{s}^{t} 2\|d(v(\theta))\| \mathrm{d} \theta\right)\left(\int A(v)(s, x) \cdot(v-J)(s, x) \mathrm{d} x\right) \mathrm{d} s
\end{aligned}
$$


where $d(v)$ is the symmetric part of $D v=\partial_{j} v_{i},\|d(v(t))\|$ is the supremum on $x$ of the spectral radius of $d(v)(t, x)$ and

$$
A(v)=\partial_{t} v+v \cdot \nabla v
$$

In the second section, we establish the equations which are satisfied by the density, the current and the energy. Then, in Section 3, we give the proof of Theorem 1.2, which is mainly based on the modulated energy method introduced in [2]. Finally, we build an example of well-prepared initial data.

\section{Equations of CONSERVATion}

\subsection{Conservation of mass and total momentum}

We define the current $\tilde{J}^{\varepsilon}$ from the density $\rho^{\varepsilon}$ using the equation

$$
\partial_{t} \rho^{\varepsilon}+\operatorname{div} \tilde{J}^{\varepsilon}=0
$$

and we obtain

$$
\tilde{J}^{\varepsilon}=J^{\varepsilon}+\frac{{ }^{\perp} x}{2 \varepsilon} \rho^{\varepsilon}
$$

with $J^{\varepsilon}$ defined by

$$
J^{\varepsilon}(t, x)=\sum_{k} \lambda_{k} \operatorname{Im}\left(h \nabla \psi_{k}^{\varepsilon}(t, x) \bar{\psi}_{k}^{\varepsilon}(t, x)\right) .
$$

Equation (2.3) is the classical form of the current when there is no magnetic field (see [10] for instance). It is important to note that the classical relation $\partial_{t} \rho^{\varepsilon}+\operatorname{div} J^{\varepsilon}=0$ does not hold here.

Remark 2.1. The difference between $J^{\varepsilon}$ and $\tilde{J}^{\varepsilon}$ must be understood like the difference between impulsion and total momentum. Indeed, $\tilde{J}^{\varepsilon}$ corresponds to a real physical quantity (cf. [4]). Assumption (1.9) can be interpreted as an hypothesis on the initial velocity or kinetic energy.

Proposition 2.2. The current $\tilde{J}^{\varepsilon}$ satisfies the following equation in the distributional sense

$$
\begin{aligned}
\partial_{t} \tilde{J}^{\varepsilon}= & -\sum_{k} \lambda_{k} \nabla:\left[\left(h \nabla \psi_{k}^{\varepsilon}+i \frac{{ }^{\perp} x}{2 \varepsilon} \psi_{k}^{\varepsilon}\right) \bar{\otimes}\left(h \nabla \bar{\psi}_{k}^{\varepsilon}-i \frac{{ }^{\perp} x}{2 \varepsilon} \bar{\psi}_{k}^{\varepsilon}\right)\right]+\frac{1}{\varepsilon}\left(-\rho^{\varepsilon} \nabla \phi^{\varepsilon}+{ }^{\perp} \tilde{J}^{\varepsilon}\right) \\
& +\sum_{k} \lambda_{k} \nabla\left(\frac{h^{2}}{4}\left(\Delta \psi_{k}^{\varepsilon} \bar{\psi}_{k}^{\varepsilon}+\Delta \bar{\psi}_{k}^{\varepsilon} \psi_{k}^{\varepsilon}+2 \nabla \psi_{k}^{\varepsilon} \cdot \nabla \bar{\psi}_{k}^{\varepsilon}\right)\right)
\end{aligned}
$$

which means

$$
\int v \cdot \partial_{t} \tilde{J}^{\varepsilon}=\sum_{k} \lambda_{k} \int \mathrm{d} v:\left(h \nabla \psi_{k}^{\varepsilon}+i \frac{{ }^{\perp} x}{2 \varepsilon} \psi_{k}^{\varepsilon}\right) \otimes\left(h \nabla \bar{\psi}_{k}^{\varepsilon}-i \frac{{ }^{\perp} x}{2 \varepsilon} \bar{\psi}_{k}^{\varepsilon}\right)+\frac{1}{\varepsilon} \int v \cdot\left(-\rho^{\varepsilon} \nabla \phi^{\varepsilon}+{ }^{\perp} \tilde{J}^{\varepsilon}\right)
$$

for all divergence free test function $v \in \mathcal{D}(] 0, T\left[\times \mathbb{R}^{2}\right)$.

Remark 2.3. We use the compact notations

$$
A \otimes B=A_{i} B_{j}, \quad(\nabla: \mathbb{B})_{i}=\partial_{j} B_{i j} \quad, \quad A \bar{\otimes} B=\frac{1}{2}(A \otimes B+\bar{A} \otimes \bar{B}) .
$$


Proof. We compute $\partial_{t} \tilde{J}^{\varepsilon}$ using (1.2) and obtain

$$
\begin{aligned}
\partial_{t} \tilde{J}^{\varepsilon}= & \sum_{k} \lambda_{k}\left[\nabla\left(\frac{h^{2}}{4}\left(\Delta \psi_{k}^{\varepsilon} \bar{\psi}_{k}^{\varepsilon}+\Delta \bar{\psi}_{k}^{\varepsilon} \psi_{k}^{\varepsilon}+2 \nabla \psi_{k}^{\varepsilon} \cdot \nabla \bar{\psi}_{k}^{\varepsilon}\right)\right)-h^{2} \nabla:\left(\nabla \psi_{k}^{\varepsilon} \bar{\otimes} \nabla \bar{\psi}_{k}^{\varepsilon}\right)\right] \\
& +\frac{1}{2 \varepsilon}{ }^{\perp} \tilde{J}^{\varepsilon}-\frac{1}{2 \varepsilon}\left({ }^{\perp} x \cdot \nabla\right) J^{\varepsilon}-\frac{1}{\varepsilon} \nabla \phi^{\varepsilon} \rho^{\varepsilon}+\frac{{ }^{\perp} x}{2 \varepsilon} \partial_{t} \rho^{\varepsilon} .
\end{aligned}
$$

Moreover, we define

$$
I_{1}=\sum_{k} \lambda_{k} \nabla:\left[\left(h \nabla \psi_{k}^{\varepsilon}+i \frac{{ }^{\perp} x}{2 \varepsilon} \psi_{k}^{\varepsilon}\right) \bar{\otimes}\left(h \nabla \bar{\psi}_{k}^{\varepsilon}-i \frac{{ }^{\perp} x}{2 \varepsilon} \bar{\psi}_{k}^{\varepsilon}\right)\right] .
$$

It follows that

$$
I_{1}=\sum_{k} \lambda_{k} \nabla:\left(h \nabla \psi_{k}^{\varepsilon} \bar{\otimes} h \nabla \bar{\psi}_{k}^{\varepsilon}\right)+\frac{1}{2} \nabla:\left(\frac{{ }^{\perp} x}{\varepsilon} \otimes J^{\varepsilon}+J^{\varepsilon} \otimes \frac{{ }^{\perp} x}{\varepsilon}\right)+\frac{1}{4 \varepsilon^{2}} \nabla:\left({ }^{\perp} x \otimes{ }^{\perp} x \rho^{\varepsilon}\right) .
$$

Then we have

$$
\begin{aligned}
I_{1} & =\sum_{k} \lambda_{k} \nabla:\left(h \nabla \psi_{k}^{\varepsilon} \bar{\otimes} h \nabla \bar{\psi}_{k}^{\varepsilon}\right)+\frac{{ }^{\perp} x}{2 \varepsilon} \operatorname{div} J^{\varepsilon}+\frac{1}{2 \varepsilon}{ }^{\perp} J^{\varepsilon}+\frac{1}{2 \varepsilon}\left({ }^{\perp} x \cdot \nabla\right) J^{\varepsilon}-\frac{1}{4 \varepsilon^{2}} x \rho^{\varepsilon}+\frac{{ }^{\perp} x}{2 \varepsilon}\left(\frac{{ }^{\perp} x}{2 \varepsilon} \cdot \nabla \rho^{\varepsilon}\right) \\
& =\sum_{k} \lambda_{k} \nabla:\left(h \nabla \psi_{k}^{\varepsilon} \bar{\otimes} h \nabla \bar{\psi}_{k}^{\varepsilon}\right)+\frac{1}{2 \varepsilon}{ }^{\perp} J^{\varepsilon}+\frac{1}{2 \varepsilon}\left({ }^{\perp} x \cdot \nabla\right) J^{\varepsilon}+\frac{1}{2 \varepsilon}{ }^{\perp}\left(\frac{{ }^{\perp} x}{2 \varepsilon}\right) \rho^{\varepsilon}-\frac{{ }^{\perp} x}{2 \varepsilon} \partial_{t} \rho^{\varepsilon},
\end{aligned}
$$

because the conservation of mass (2.1) implies

$$
\frac{{ }^{\perp} x}{2 \varepsilon}\left(\operatorname{div} J^{\varepsilon}+\frac{{ }^{\perp} x}{2 \varepsilon} \cdot \nabla \rho^{\varepsilon}\right)=-\frac{{ }^{\perp} x}{2 \varepsilon} \partial_{t} \rho^{\varepsilon}
$$

This computation gives us equation (2.4) from (2.5) because

$$
\begin{aligned}
& \partial_{t} \tilde{J}^{\varepsilon}+\sum_{k} \lambda_{k} \nabla:\left[\left(h \nabla \psi_{k}^{\varepsilon}+i \frac{{ }^{\perp} x}{2 \varepsilon} \psi_{k}^{\varepsilon}\right) \bar{\otimes}\left(h \nabla \bar{\psi}_{k}^{\varepsilon}-i \frac{{ }^{\perp} x}{2 \varepsilon} \bar{\psi}_{k}^{\varepsilon}\right)\right] \\
& =\partial_{t} \tilde{J}^{\varepsilon}+\sum_{k} \lambda_{k} \nabla:\left(h \nabla \psi_{k}^{\varepsilon} \bar{\otimes} h \nabla \bar{\psi}_{k}^{\varepsilon}\right)+\frac{1}{2 \varepsilon}^{\perp} J^{\varepsilon}+\frac{1}{2 \varepsilon}\left({ }^{\perp} x \cdot \nabla\right) J^{\varepsilon}+\frac{1}{2 \varepsilon}^{\perp}\left(\frac{{ }^{\perp} x}{2 \varepsilon}\right) \rho^{\varepsilon}-\frac{{ }^{\perp} x}{2 \varepsilon} \partial_{t} \rho^{\varepsilon} \\
& =\frac{1}{\varepsilon}\left(-\rho^{\varepsilon} \nabla \phi^{\varepsilon}+{ }^{\perp} \tilde{J}^{\varepsilon}\right)+\sum_{k} \lambda_{k} \nabla\left(\frac{h^{2}}{4}\left(\Delta \psi_{k}^{\varepsilon} \bar{\psi}_{k}^{\varepsilon}+\Delta \bar{\psi}_{k}^{\varepsilon} \psi_{k}^{\varepsilon}+2 \nabla \psi_{k}^{\varepsilon} \cdot \nabla \bar{\psi}_{k}^{\varepsilon}\right)\right) .
\end{aligned}
$$




\subsection{Conservation of energy}

The energy of the system is the following

$$
\tilde{H}^{\varepsilon}(t)=\sum_{k} \lambda_{k} \frac{1}{2} \varepsilon \int_{\mathbb{R}^{2}}\left|h \nabla \psi_{k}^{\varepsilon}(t, x)+i \frac{{ }^{\perp} x}{2 \varepsilon} \psi_{k}^{\varepsilon}(t, x)\right|^{2} \mathrm{~d} x+\frac{1}{2} \int_{\mathbb{R}^{2}}\left|\nabla \phi^{\varepsilon}(t, x)\right|^{2} \mathrm{~d} x
$$

and satisfies:

Proposition 2.4. Energy $H^{\varepsilon}$ remains constant with respect to time.

The proof of this proposition is given in Section 5 .

\section{Proof of Theorem 1.2}

\subsection{Definition of the modulated energy}

Proposition 3.1. For all divergence free test function $v$, we define a modulated energy

$$
\tilde{H}_{v}^{\varepsilon}(t)=\sum_{k} \lambda_{k} \frac{\varepsilon}{2} \int\left(h \nabla \psi_{k}^{\varepsilon}+\frac{i}{2 \varepsilon}{ }^{\perp} x \psi_{k}^{\varepsilon}-i v \psi_{k}^{\varepsilon}\right)\left(h \nabla \bar{\psi}_{k}^{\varepsilon}-\frac{i}{2 \varepsilon}{ }^{\perp} x \bar{\psi}_{k}^{\varepsilon}+i v \bar{\psi}_{k}^{\varepsilon}\right)+\frac{1}{2} \int\left|\nabla\left(\phi^{\varepsilon}-\Psi\right)\right|^{2}
$$

with ${ }^{\perp} \nabla \Psi=v$. The function $\tilde{H}_{v}^{\varepsilon}(t)$ satisfies

$$
\begin{aligned}
\frac{\mathrm{d}}{\mathrm{d} t} \tilde{H}_{v}^{\varepsilon}(t)= & -\varepsilon \int \sum_{k} \lambda_{k} \mathrm{~d} v:\left(h \nabla \psi_{k}^{\varepsilon}+\frac{i}{2 \varepsilon}{ }^{\perp} x \psi_{k}^{\varepsilon}-i v \psi_{k}^{\varepsilon}\right) \otimes\left(h \nabla \bar{\psi}_{k}^{\varepsilon}-\frac{i}{2 \varepsilon}{ }^{\perp} x \bar{\psi}_{k}^{\varepsilon}+i v \bar{\psi}_{k}^{\varepsilon}\right) \\
& +\int \mathrm{d} v: \nabla\left(\phi^{\varepsilon}-\Psi\right) \otimes \nabla\left(\phi^{\varepsilon}-\Psi\right) \\
& +\varepsilon \int A(v) \cdot\left(\rho^{\varepsilon} v-\tilde{J}^{\varepsilon}\right)+\int \theta^{\varepsilon} v \nabla \phi^{\varepsilon}+\int A(v) \cdot\left(v+{ }^{\perp} \nabla \phi^{\varepsilon}\right) .
\end{aligned}
$$

Proof. Differentiating the modulated energy (3.1) with respect to time, we obtain

$$
\begin{aligned}
\frac{\mathrm{d}}{\mathrm{d} t} \tilde{H}_{v}^{\varepsilon}(t)= & \sum_{k} \lambda_{k} \frac{\mathrm{d}}{\mathrm{d} t}\left[\int \left(\frac{\varepsilon}{2} h^{2}\left|\nabla \psi_{k}^{\varepsilon}+\frac{i}{2 \varepsilon}{ }^{\perp} x \psi_{k}^{\varepsilon}\right|^{2}-\frac{h \varepsilon}{2 i} v \cdot\left(\nabla \psi_{k}^{\varepsilon} \bar{\psi}_{k}^{\varepsilon}-\psi_{k}^{\varepsilon} \nabla \bar{\psi}_{k}^{\varepsilon}\right)-v \cdot \frac{{ }^{\perp} x}{2} \rho^{\varepsilon}\right.\right. \\
& \left.\left.+\frac{\varepsilon}{2}|v|^{2} \psi_{k}^{\varepsilon} \bar{\psi}_{k}^{\varepsilon}+\frac{1}{2}\left|\nabla \phi^{\varepsilon}\right|^{2}-\nabla \phi^{\varepsilon} \cdot \nabla \Psi+\frac{1}{2}|\nabla \Psi|^{2}\right)\right] .
\end{aligned}
$$

It can be expressed in term of the energy $\tilde{H}^{\varepsilon}$ in the following way

$\frac{\mathrm{d}}{\mathrm{d} t} \tilde{H}_{v}^{\varepsilon}(t)=\frac{\mathrm{d}}{\mathrm{d} t} \tilde{H}^{\varepsilon}+\sum_{k} \lambda_{k} \frac{\mathrm{d}}{\mathrm{d} t} \int\left(-\frac{h \varepsilon}{2 i} v \cdot\left(\nabla \psi_{k}^{\varepsilon} \bar{\psi}_{k}^{\varepsilon}-\psi_{k}^{\varepsilon} \nabla \bar{\psi}_{k}^{\varepsilon}\right)-v \cdot \frac{{ }^{\perp} x}{2} \rho^{\varepsilon}+\frac{\varepsilon}{2}|v|^{2} \psi_{k}^{\varepsilon} \bar{\psi}_{k}^{\varepsilon}-\nabla \phi^{\varepsilon} \cdot \nabla \Psi+\frac{1}{2}|\nabla \Psi|^{2}\right)$. 
Thanks to Proposition 2.4, we know that the energy is conserved. Therefore, we obtain the following simplified form for the time derivative of the modulated energy $\tilde{H}_{v}^{\varepsilon}$

$$
\begin{aligned}
\frac{\mathrm{d}}{\mathrm{d} t} \tilde{H}_{v}^{\varepsilon}(t)= & \frac{\mathrm{d}}{\mathrm{d} t} \int-\varepsilon \tilde{J}^{\varepsilon} \cdot v+\frac{\varepsilon}{2}|v|^{2} \rho^{\varepsilon}-\nabla \phi^{\varepsilon} \cdot \nabla \Psi+\frac{1}{2}|\nabla \Psi|^{2} \\
= & \int \frac{\varepsilon}{2}|v|^{2} \partial_{t} \rho^{\varepsilon}+\frac{\varepsilon}{2} \int \rho^{\varepsilon} \partial_{t}|v|^{2}-\varepsilon\left\langle v ; \partial_{t} \tilde{J}^{\varepsilon}\right\rangle_{\mathcal{D}, \mathcal{D}^{\prime}}-\varepsilon \int \tilde{J}^{\varepsilon} \cdot \partial_{t} v \\
& +\frac{1}{2} \int \partial_{t}|v|^{2}-\int \nabla \phi^{\varepsilon} \cdot \partial_{t} \nabla \Psi-\left\langle\nabla \Psi ; \partial_{t} \nabla \phi^{\varepsilon}\right\rangle_{\mathcal{D}, \mathcal{D}^{\prime}}
\end{aligned}
$$

since ${ }^{\perp} \nabla \Psi=v$.

We compute each term of this identity. First of all

$$
\begin{aligned}
-\left\langle\nabla \Psi ; \partial_{t} \nabla \phi^{\varepsilon}\right\rangle_{\mathcal{D}, \mathcal{D}^{\prime}} & =\int \partial_{t} \Delta \phi^{\varepsilon} \Psi=-\int \partial_{t} \rho^{\varepsilon} \Psi \\
& =\int \operatorname{div} \tilde{J}^{\varepsilon} \Psi=-\int \tilde{J}^{\varepsilon} \cdot \nabla \Psi
\end{aligned}
$$

Furthermore, because of equation (2.4) satisfied by $\partial_{t} \tilde{J}^{\varepsilon}$, we obtain

$$
\begin{aligned}
I_{3}=-\varepsilon\left\langle v ; \partial_{t} \tilde{J}^{\varepsilon}\right\rangle_{\mathcal{D}, \mathcal{D}^{\prime}}= & -\varepsilon \int \sum_{k} \lambda_{k} \mathrm{~d} v:\left(h \nabla \psi_{k}^{\varepsilon}+\frac{i}{2 \varepsilon}{ }^{\perp} x \psi_{k}^{\varepsilon}\right) \otimes\left(h \nabla \bar{\psi}_{k}^{\varepsilon}-\frac{i}{2 \varepsilon}{ }^{\perp} x \bar{\psi}_{k}^{\varepsilon}\right) \\
& +\int \rho^{\varepsilon} v \cdot \nabla \phi^{\varepsilon}-\int v \cdot{ }^{\perp} \tilde{J}^{\varepsilon},
\end{aligned}
$$

that we transform, using ${ }^{\perp} \nabla \Psi=v$ and the Poisson equation (1.3), into

$$
\begin{aligned}
I_{3}= & -\varepsilon \int \sum_{k} \lambda_{k} \mathrm{~d} v:\left(h \nabla \psi_{k}^{\varepsilon}+\frac{i}{2 \varepsilon}{ }^{\perp} x \psi_{k}^{\varepsilon}\right) \otimes\left(h \nabla \bar{\psi}_{k}^{\varepsilon}-\frac{i}{2 \varepsilon}{ }^{\perp} x \bar{\psi}_{k}^{\varepsilon}\right) \\
& -\int \Delta \phi^{\varepsilon} v \cdot \nabla \phi^{\varepsilon}+\int \theta^{\varepsilon} v \nabla \phi^{\varepsilon}+\int \tilde{J}^{\varepsilon} \cdot \nabla \Psi .
\end{aligned}
$$

Moreover,

$$
\begin{aligned}
-\int \Delta \phi^{\varepsilon} v \cdot \nabla \phi^{\varepsilon} & =\int \partial_{j} \partial_{j} \phi^{\varepsilon} v_{i} \partial_{i} \phi^{\varepsilon}=\int \partial_{j} \phi^{\varepsilon} \partial_{j} v_{i} \partial_{i} \phi^{\varepsilon}+\partial_{j} \phi^{\varepsilon} v_{i} \partial_{j} \partial_{i} \phi^{\varepsilon} \\
& =\int \mathrm{d} v: \nabla \phi^{\varepsilon} \otimes \nabla \phi^{\varepsilon} .
\end{aligned}
$$

We have then

$$
\begin{aligned}
I_{3}= & -\varepsilon \int \sum_{k} \lambda_{k} \mathrm{~d} v:\left(h \nabla \psi_{k}^{\varepsilon}+\frac{i}{2 \varepsilon}{ }^{\perp} x \psi_{k}^{\varepsilon}\right) \otimes\left(h \nabla \bar{\psi}_{k}^{\varepsilon}-\frac{i}{2 \varepsilon}{ }^{\perp} x \bar{\psi}_{k}^{\varepsilon}\right) \\
& +\int \mathrm{d} v: \nabla \phi^{\varepsilon} \otimes \nabla \phi^{\varepsilon}+\int \theta^{\varepsilon} v \nabla \phi^{\varepsilon}+\int \tilde{J}^{\varepsilon} \cdot \nabla \Psi .
\end{aligned}
$$


To obtain equation (3.2), we must study the modulation by $v={ }^{\perp} \nabla \Psi$ and by $\nabla \Psi$ in the two terms of energy $\tilde{H}^{\varepsilon}$ given by (2.6). Let us study the first term

$$
\begin{aligned}
& -\varepsilon \int \sum_{k} \lambda_{k} \mathrm{~d} v:\left(h \nabla \psi_{k}^{\varepsilon}+\frac{i}{2 \varepsilon}{ }^{\perp} x \psi_{k}^{\varepsilon}\right) \otimes\left(h \nabla \bar{\psi}_{k}^{\varepsilon}-\frac{i}{2 \varepsilon}{ }^{\perp} x \bar{\psi}_{k}^{\varepsilon}\right) \\
& =-\varepsilon \int \sum_{k} \lambda_{k} D v:\left[\left(h \nabla \psi_{k}^{\varepsilon}+\frac{i}{2 \varepsilon}{ }^{\perp} x \psi_{k}^{\varepsilon}\right) \bar{\otimes}\left(h \nabla \bar{\psi}_{k}^{\varepsilon}-\frac{i}{2 \varepsilon}{ }^{\perp} x \bar{\psi}_{k}^{\varepsilon}\right)\right] \\
& =-\varepsilon \int \sum_{k} \lambda_{k} D v:\left[\left(h \nabla \psi_{k}^{\varepsilon}+\frac{i}{2 \varepsilon}{ }^{\perp} x \psi_{k}^{\varepsilon}-i v \psi_{k}^{\varepsilon}\right) \bar{\otimes}\left(h \nabla \bar{\psi}_{k}^{\varepsilon}-\frac{i}{2 \varepsilon}{ }^{\perp} x \bar{\psi}_{k}^{\varepsilon}+i v \bar{\psi}_{k}^{\varepsilon}\right)\right] \\
& -\varepsilon \int D v: \tilde{J}^{\varepsilon} \otimes v-\varepsilon \int D v: v \otimes \tilde{J}^{\varepsilon}+\varepsilon \int \rho^{\varepsilon} D v: v \otimes v .
\end{aligned}
$$

The conservation of mass ensures that

$$
\begin{aligned}
-\varepsilon \int D v: v \otimes \tilde{J}^{\varepsilon} & =-\varepsilon \int \partial_{j} v_{i} \tilde{J}_{j}^{\varepsilon} v_{i}=\frac{\varepsilon}{2} \int \operatorname{div} \tilde{J}^{\varepsilon}|v|^{2} \\
& =-\frac{\varepsilon}{2} \int \partial_{t} \rho^{\varepsilon}|v|^{2}
\end{aligned}
$$

Now, we can use the results shown by Brenier in [2] to obtain

$$
\begin{aligned}
\int \mathrm{d} v: \nabla \phi^{\varepsilon} \otimes \nabla \phi^{\varepsilon}= & \int \mathrm{d} v: \nabla\left(\phi^{\varepsilon}-\Psi\right) \otimes \nabla\left(\phi^{\varepsilon}-\Psi\right)+\int D v: \nabla \Psi \otimes \nabla \phi^{\varepsilon} \\
& +\int D v: \nabla \phi^{\varepsilon} \otimes \nabla \Psi-\int D v: \nabla \Psi \otimes \nabla \Psi .
\end{aligned}
$$

In [2], it is also shown that

$$
\frac{\varepsilon}{2} \int \rho^{\varepsilon} \partial_{t}|v|^{2}-\varepsilon \int \tilde{J}^{\varepsilon} \cdot \partial_{t} v-\varepsilon \int D v: \tilde{J}^{\varepsilon} \otimes v+\varepsilon \int \rho^{\varepsilon} D v: v \otimes v=\varepsilon \int A(v) \cdot\left(\rho^{\varepsilon} v-\tilde{J}^{\varepsilon}\right),
$$

$\frac{1}{2} \int \partial_{t}|v|^{2}-\int \nabla \phi^{\varepsilon} \cdot \partial_{t} \Psi+\int D v: \nabla \Psi \otimes \nabla \phi^{\varepsilon}+\int D v: \nabla \phi^{\varepsilon} \otimes \nabla \Psi-\int D v: \nabla \Psi \otimes \nabla \Psi=\int A(v) \cdot\left(v+{ }^{\perp} \nabla \phi^{\varepsilon}\right)$

so we obtain (3.2) introducing the modulation in (3.3).

Remark 3.2. Since the functions $\Psi$ and $v$ are test functions with compact support, the integrations by parts do not give any boundary term. And they are well defined because $\rho^{\varepsilon}(t, \cdot), \partial_{t} \rho^{\varepsilon}(t, \cdot), \operatorname{div} \tilde{J}^{\varepsilon}(t, \cdot)$ are in $L^{1}\left(\mathbb{R}^{2}\right)$, $\tilde{J}^{\varepsilon}(t, \cdot) \in L^{1}\left(\mathbb{R}^{2}, \mathbb{R}^{2}\right), \nabla \phi^{\varepsilon}(t, \cdot) \in L^{\infty}\left(\mathbb{R}^{2}, \mathbb{R}^{2}\right)$ and $\Delta \phi^{\varepsilon}(t, \cdot) \in L^{1}\left(\mathbb{R}^{2}\right)$.

\subsection{Convergence to the Euler equations}

We prove in this section that $\left({ }^{\perp} \nabla \phi^{\varepsilon}\right)$ converges in $C^{0}\left([0, T], \mathcal{D}^{\prime}\left(\mathbb{R}^{2}, \mathbb{R}^{2}\right)\right)$ to a dissipative solution of the Euler equations. We first establish a result about the convergence and then we perform some estimates involving the modulated energy to identify the limit. 


\subsubsection{Convergence}

Proposition 3.3. The sequence $\left(\rho^{\varepsilon}\right)$ is compact in $C^{0}\left([0, T] ; \mathcal{D}^{\prime}\left(\mathbb{R}^{2}\right)\right)$, $\left(\tilde{J}^{\varepsilon}\right)$ is bounded in $\mathcal{D}^{\prime}(] 0, T\left[\times \mathbb{R}^{2}\right),\left(H_{v}^{\varepsilon}\right)$ converges, up to a subsequence, in $L^{\infty}([0, T])-w *$ and $\left(\nabla \phi^{\varepsilon}\right)$ converges in $C^{0}\left([0, T] ; \mathcal{D}^{\prime}\left(\mathbb{R}^{2} ; \mathbb{R}^{2}\right)\right)$.

Let us first note that the conservation of energy implies that $\tilde{H}^{\varepsilon}(t)=\tilde{H}^{\varepsilon}(0)$ and assumptions $(1.9)$ and (1.8) imply that $\left(\tilde{H}^{\varepsilon}(0)\right)$ converges. Thus, there exists $C$ independent on $t$ and $\varepsilon$ such that $\tilde{H}^{\varepsilon}(t) \leq C$. This inequality implies that $\left(\nabla \phi^{\varepsilon}\right)$ is bounded independently on $\varepsilon$ in $L^{\infty}\left([0, T] ; L^{2}\left(\mathbb{R}^{2}, \mathbb{R}^{2}\right)\right)$ and then $\left(\rho^{\varepsilon}-\theta^{\varepsilon}\right)$ is bounded in $L^{\infty}\left([0, T] ; H^{-1}\left(\mathbb{R}^{2}\right)\right)$ since, for all test function $v$, we have

$$
\begin{aligned}
\left|\int\left(\rho^{\varepsilon}(t, x)-\theta^{\varepsilon}(t, x)\right) v(x) \mathrm{d} x\right| & =\left|-\int \Delta \phi^{\varepsilon}(t, x) v(x) \mathrm{d} x\right| \\
& \leq C\|\nabla v\|_{L^{2}\left(\mathbb{R}^{2}\right)}
\end{aligned}
$$

Remark 3.4. The density $\rho^{\varepsilon}$ given by (1.1) satisfies $\int \rho^{\varepsilon}(t, x) \mathrm{d} x=\int \rho^{\varepsilon}(0, x) \mathrm{d} x$ but $\int \rho^{\varepsilon}(0, x) \mathrm{d} x \longrightarrow \infty$ when $\varepsilon$ goes to zero.

Let us now show that $\left(\varepsilon^{\frac{1}{2}} \tilde{J}^{\varepsilon}\right)$ is bounded in $L^{\infty}\left([0, T] ;\left(W^{1,4}\left(\mathbb{R}^{2}, \mathbb{R}^{2}\right) \cap L^{2}\left(\mathbb{R}^{2}, \mathbb{R}^{2}\right)\right)^{\prime}\right)$.

Indeed, since $\tilde{J}^{\varepsilon}=\sum_{k} \lambda_{k} \operatorname{Im}\left[\left(h \nabla \psi_{k}^{\varepsilon}+i \frac{{ }^{\perp} x}{2 \varepsilon} \psi_{k}^{\varepsilon}\right) \bar{\psi}_{k}^{\varepsilon}\right]$, we have

$$
\begin{aligned}
\left|\int \varepsilon^{\frac{1}{2}} \tilde{J}^{\varepsilon} \cdot v \mathrm{~d} x\right| & \leq\left(\varepsilon \int \sum_{k} \lambda_{k}\left|h \nabla \psi_{k}^{\varepsilon}+i \frac{{ }^{\perp} x}{2 \varepsilon} \psi_{k}^{\varepsilon}\right|^{2}\right)^{\frac{1}{2}}\left(\int \sum_{k} \lambda_{k}\left|\psi_{k}^{\varepsilon}\right|^{2}|v|^{2}\right)^{\frac{1}{2}} \\
& \leq C\left(\|v\|_{W^{1,4}}^{2}+\|v\|_{L^{2}}^{2}\right)^{\frac{1}{2}}
\end{aligned}
$$

Furthermore, the Poisson equation (1.3), which links $\rho^{\varepsilon}$ and $\phi^{\varepsilon}$, implies that $\left(\rho^{\varepsilon}-\theta^{\varepsilon}\right) \nabla \phi^{\varepsilon}$ is bounded in $L^{\infty}\left([0, T] ;\left(W^{m, p}\left(\mathbb{R}^{2}, \mathbb{R}^{2}\right)\right)^{\prime}\right)$ with $m-1>\frac{2}{p}$ because for all $g \in W^{m, p}\left(\mathbb{R}^{2}, \mathbb{R}^{2}\right)$

$$
\begin{aligned}
&\left|\int g(x) \cdot\left(\rho^{\varepsilon}(t, x)-\theta^{\varepsilon}(t, x)\right) \nabla \phi^{\varepsilon}(t, x) \mathrm{d} x\right|=\left|-\int \Delta \phi^{\varepsilon}(t, x) g(x) \cdot \nabla \phi^{\varepsilon}(t, x) \mathrm{d} x\right| \\
&= \mid \int\left(\nabla \phi^{\varepsilon}(t, x) \cdot \nabla\right) g(x) \cdot \nabla \phi^{\varepsilon}(t, x) \mathrm{d} x \\
&-\int \frac{1}{2}\left|\nabla \phi^{\varepsilon}(t, x)\right|^{2} \operatorname{div} g(x) \mathrm{d} x \mid \\
& \leq C\|g\|_{C^{1}\left(\mathbb{R}^{2}\right)} \leq C\|g\|_{W^{m, p}},
\end{aligned}
$$

recalling that $m-1 \geq 2 / p$. Additionally, we have

$$
\partial_{t}\left(\rho^{\varepsilon}-\varepsilon^{\perp} \nabla \cdot \tilde{J}^{\varepsilon}\right)+^{\perp} \nabla \cdot\left(\rho^{\varepsilon} \nabla \phi^{\varepsilon}\right)=\sum_{k} \lambda_{k} \varepsilon^{\perp} \nabla \cdot\left(\nabla_{x}:\left[\left(h \nabla \psi_{k}^{\varepsilon}+i \frac{{ }^{\perp} x}{2 \varepsilon} \psi_{k}^{\varepsilon}\right) \bar{\otimes}\left(h \nabla \bar{\psi}_{k}^{\varepsilon}-i \frac{{ }^{\perp} x}{2 \varepsilon} \bar{\psi}_{k}^{\varepsilon}\right)\right]\right) .
$$

Since $\left(\left(\rho^{\varepsilon}-\theta^{\varepsilon}\right) \nabla \phi^{\varepsilon}\right)$ is bounded in $L^{\infty}\left([0, T] ;\left(W^{m, p}\left(\mathbb{R}^{2}, \mathbb{R}^{2}\right)\right)^{\prime}\right)$ and since the energy is conserved, identity (3.4) implies that $\left(\partial_{t}\left(\rho^{\varepsilon}-\varepsilon^{\perp} \nabla . \tilde{J}^{\varepsilon}\right)\right)$ is bounded in $L^{\infty}\left([0, T] ;\left(W^{m+1, p}\left(\mathbb{R}^{2}\right) \cap H^{1}\left(\mathbb{R}^{2}\right)\right)^{\prime}\right)$ and then, using Aubin's 
Lemma locally ( $c f$. appendices of [7] and [6]), $\left(\rho^{\varepsilon}-\varepsilon^{\perp} \nabla \cdot \tilde{J}^{\varepsilon}\right)$ is compact in $C^{0}\left([0, T] ; \mathcal{D}^{\prime}\left(\mathbb{R}^{2}\right)\right)$. Since $\varepsilon^{\perp} \nabla \cdot \tilde{J}^{\varepsilon}=$ $O\left(\varepsilon^{\frac{1}{2}}\right)$ in $C^{0}\left([0, T] ; \mathcal{D}^{\prime}\left(\mathbb{R}^{2}\right)\right)$ we show that $\left(\rho^{\varepsilon}\right)$ is compact in $C^{0}\left([0, T] ; \mathcal{D}^{\prime}\left(\mathbb{R}^{2}\right)\right)$. Since $\rho^{\varepsilon}=\theta^{\varepsilon}-\Delta \phi^{\varepsilon},\left(\nabla \phi^{\varepsilon}\right)$ is also compact and then, up to a subsequence, $\left(\nabla \phi^{\varepsilon}\right)$ converges in $C^{0}\left([0, T] ; \mathcal{D}^{\prime}\left(\mathbb{R}^{2}, \mathbb{R}^{2}\right)\right)$. The modulated energy $\left(\tilde{H}_{v}^{\varepsilon}\right)$ is bounded in $L^{\infty}([0, T])$ and then, up to a subsequence, converges in $L^{\infty}([0, T])-w *$.

This concludes the proof of Proposition 3.3.

\subsubsection{Identification of the limit}

Inequality (3.2) can be written in a weak form in the following way

$$
\begin{aligned}
-\int \tilde{H}_{v}^{\varepsilon}(t) z^{\prime}(t) \mathrm{d} t-z(0) \tilde{H}_{v}^{\varepsilon}(0) \leq & \int 2\|\mathrm{~d} v\| \tilde{H}_{v}^{\varepsilon}(t) z(t) \mathrm{d} t+\int\left[\varepsilon A(v)\left(\rho^{\varepsilon} v-\tilde{J}^{\varepsilon}\right)(t, x)\right] z(t) \mathrm{d} t \mathrm{~d} x \\
& +\int\left[A(v)\left(v+{ }^{\perp} \nabla \phi^{\varepsilon}\right)(t, x)+v \theta^{\varepsilon} \nabla \phi^{\varepsilon}(t, x)\right] z(t) \mathrm{d} t \mathrm{~d} x,
\end{aligned}
$$

where $z$ is a test function belonging to $\mathcal{D}([0, T[)$.

As soon as $\varepsilon$ is small enough, $\theta^{\varepsilon}=1$ on the support of $v$. We can pass to the limit because $\left(\tilde{H}_{v}^{\varepsilon}\right)$ converges in $L^{\infty}([0, T])-w *,\left(\rho^{\varepsilon}\right)$ and $\left(\tilde{J}^{\varepsilon}\right)$ are bounded in the sense of distributions and $\left(\nabla \phi^{\varepsilon}\right)$ converges in $C^{0}\left([0, T] ; \mathcal{D}^{\prime}\left(\mathbb{R}^{2}, \mathbb{R}^{2}\right)\right)$.

Then for all test function $z \in \mathcal{D}([0, T[)$, we have

$$
-\int \tilde{H}_{v}(t) z^{\prime}(t) \mathrm{d} t-z(0) \tilde{H}_{v, 0} \leq \int 2\|\mathrm{~d} v\| \tilde{H}_{v}(t) z(t) \mathrm{d} t+\int A(v)\left(v+{ }^{\perp} \nabla \phi\right)(t, x) z(t) \mathrm{d} t \mathrm{~d} x,
$$

with $\tilde{H}_{v, 0}=\lim _{\varepsilon \rightarrow 0} \tilde{H}_{v}^{\varepsilon}(0)$.

Using Gronwall's Lemma, we obtain

$$
\begin{aligned}
\|^{\perp} \nabla(\phi)-{ }^{\perp} \nabla(\Psi)||_{L^{2}\left(\mathbb{R}^{2}\right)}^{2} \leq & 2 \tilde{H}_{v}(t) \leq 2 \tilde{H}_{0, v} \exp \left(\int_{0}^{t} 2\|\mathrm{~d} v(\theta)\| \mathrm{d} \theta\right) \\
& +2 \int_{0}^{t} \exp \left(\int_{s}^{t} 2\|\mathrm{~d} v(\theta)\| \mathrm{d} \theta\right)\left(\int A(v)(s, x) \cdot\left(v+{ }^{\perp} \nabla \phi\right)(s, x) \mathrm{d} x\right) \mathrm{d} s .
\end{aligned}
$$

Since assumptions $(1.8,1.9)$ hold, we have $\tilde{H}_{v, 0}=\lim _{\varepsilon \rightarrow 0} \int \frac{1}{2}\left|\nabla \phi^{\varepsilon}(0, x)-\nabla \Psi(0, x)\right|^{2} \mathrm{~d} x$.

Moreover, we have

$$
\begin{aligned}
\left\|^{\perp} \nabla(\phi)-{ }^{\perp} \nabla(\Psi)\right\|_{L^{2}\left(\mathbb{R}^{2}\right)}^{2} & =\|\nabla(\phi)-\nabla(\Psi)\|_{L^{2}\left(\mathbb{R}^{2}\right)}^{2} \leq \liminf \left\|\nabla\left(\phi^{\varepsilon}\right)-\nabla(\Psi)\right\| \\
& \leq \liminf \tilde{H}_{v}^{\varepsilon}(t)=\tilde{H}_{v}(t) .
\end{aligned}
$$

And then, if $\int\left|\nabla \phi^{\varepsilon}(0, x)-\nabla \phi_{0}(x)\right|^{2} \mathrm{~d} x \rightarrow 0,-{ }^{\perp} \nabla \phi$ is a dissipative solution of the Euler equations with $J^{0}=$ $-{ }^{\perp} \nabla \phi_{0}$ as an initial condition.

\subsection{Construction of an example of well-prepared initial data}

\subsubsection{Condition on the potential energy}

To build an initial condition satisfying assumptions $(1.8,1.9)$, we consider the case when $\omega_{0}=$ curl $J^{0}$ belongs to $\mathcal{D}\left(\mathbb{R}^{2} ;\right]-1,+1[)$ with $\int \omega_{0}=0$, and we pick up $\varepsilon$ small enough so that $\theta^{\varepsilon}=1$ on the support of $\omega_{0}$. Let $\phi_{0}$ 
be a solution in $\mathbb{R}^{2}$ of

$$
\Delta \phi_{0}=\omega_{0}
$$

such that $J^{0}=-{ }^{\perp} \nabla \phi_{0}$. Assumption (1.8) can be written as

$$
\int\left|\nabla \phi^{\varepsilon}(0, x)-\nabla \phi_{0}(x)\right|^{2} \mathrm{~d} x \rightarrow 0
$$

We also have to assume the global neutrality at the initial time which can be written

$$
\int \theta^{\varepsilon}(x) \mathrm{d} x=\int \rho^{\varepsilon}(0, x) \mathrm{d} x=0 .
$$

To estimate $\int\left|\nabla \phi^{\varepsilon}(0, x)-\nabla \phi_{0}(x)\right|^{2} \mathrm{~d} x$, we use a result from [1], stating that, if $\Delta \phi=f$ with $\int f=0$, then $\|\nabla \phi\|_{L^{2}} \leq C\left(\||| x \mid f\|_{L^{1}}+\|f\|_{L^{2}}\right)$.

Write $f(x)=\theta^{\varepsilon}(x)-\rho^{\varepsilon}(0, x)-\omega_{0}(x)$. If the global neutrality is satisfied, we have $\int f(x) \mathrm{d} x=0$ and then

$$
\int\left|\nabla \phi^{\varepsilon}(0, x)-\nabla \phi_{0}(x)\right|^{2} \mathrm{~d} x \leq C\left(\left|\|x|f|\|_{L^{1}}+\|f\|_{L^{2}}\right)^{2}\right.
$$

Let $C_{k}$ be the elementary box defined by $\left.C_{k}=\right] y_{k}-\eta ; y_{k}+\eta\left[{ }^{2}\right.$ with $\left.y_{k}=\left(k_{1} \eta, k_{2} \eta\right), k \in \mathbb{Z}^{2} \cap\right]-\frac{1}{\eta \sigma^{\varepsilon}} ; \frac{1}{\eta \sigma^{\varepsilon}}\left[{ }^{2}, \sigma^{\varepsilon}\right.$ being such that the support of $\theta^{\varepsilon}$ is included in $\left.I_{\sigma^{\varepsilon}}=\right]-\frac{1}{\sigma^{\varepsilon}} ; \frac{1}{\sigma^{\varepsilon}}\left[{ }^{2}\right.$ and $\lambda_{k}=\eta^{2}=\left|C_{k}\right|$.

Let $\theta^{\varepsilon, \eta}$ and $\omega_{0}^{\eta}$ be defined by

$$
\begin{array}{r}
\forall x \in C_{k} \theta^{\varepsilon, \eta}(x)=\frac{1}{\left|C_{k}\right|} \int_{C_{k}} \theta^{\varepsilon}(y) \mathrm{d} y, \\
\omega_{0}^{\eta}(x)=\frac{1}{\left|C_{k}\right|} \int_{C_{k}} \omega_{0}(y) \mathrm{d} y .
\end{array}
$$

We take

$$
\left\{\begin{array}{l}
\psi_{k}^{\varepsilon, h}(x)=(2 \pi h)^{-\frac{2}{4}} \sqrt{\left(\theta^{\varepsilon, \eta}-\omega_{0}^{\eta}\right)}\left(y_{k}\right) \exp \left(-\frac{\left(x-y_{k}\right)^{2}}{2 h}\right) \exp \left(i \frac{{ }^{\perp} y_{k} \cdot x}{\varepsilon h}\right) \text { if }|x| \leq \frac{1}{\sigma^{\varepsilon}} \\
\psi_{k}^{\varepsilon, h}(x)=0 \text { elsewhere. }
\end{array}\right.
$$

Let us remark that, since $\theta^{\varepsilon, \eta}(x)=1$ on the support of $\omega_{0}$, the function $\sqrt{\left(\theta^{\varepsilon, \eta}-\omega_{0}^{\eta}\right)}$ is well defined, since we assumed $\left|\omega_{0}\right|<1$. We then get

$$
\left\{\begin{array}{l}
\rho_{0}^{\varepsilon, h}(x)=\sum_{k} \lambda_{k}(2 \pi h)^{-\frac{2}{2}}\left(\theta^{\varepsilon, \eta}-\omega_{0}^{\eta}\right)\left(y_{k}\right) \exp \left(-\frac{\left(x-y_{k}\right)^{2}}{h}\right) \text { if }|x| \leq \frac{1}{\sigma^{\varepsilon}} \\
\rho_{0}^{\varepsilon, h}(x)=0 \text { elsewhere. }
\end{array}\right.
$$


We have $\int_{\mathbb{R}^{2}} \rho_{0}^{\varepsilon, h}(x) \mathrm{d} x=\int \theta^{\varepsilon}(x) \mathrm{d} x$ and then $\int f(x) \mathrm{d} x=0$. Indeed,

$$
\begin{aligned}
\int_{\mathbb{R}^{2}} \rho_{0}^{\varepsilon, h}(x) \mathrm{d} x & =\sum_{k} \lambda_{k}\left(\theta^{\varepsilon, \eta}-\omega_{0}^{\eta}\right)\left(y_{k}\right)=\int_{I_{\sigma^{\varepsilon}}}\left(\theta^{\varepsilon}-\omega_{0}\right)(y) \mathrm{d} y \\
& =\int_{\mathbb{R}^{2}}\left(\theta^{\varepsilon}-\omega_{0}\right)(y) \mathrm{d} y=\int_{\mathbb{R}^{2}} \theta^{\varepsilon}(y) \mathrm{d} y .
\end{aligned}
$$

We want to show that ||$|x| f\left\|_{L^{1}}+\right\| f \|_{L^{2}} \longrightarrow 0$ when both $h$ and $\varepsilon$ go to zero. In order to obtain this result, we perform the following computation.

First of all, we have

$$
\left|\rho_{0}^{\varepsilon, h}(x)-\int_{I_{\sigma} \varepsilon}(2 \pi h)^{-1}\left(\theta^{\varepsilon, \eta}-\omega_{0}^{\eta}\right)(y) \exp \left(-\frac{(x-y)^{2}}{h}\right) \mathrm{d} y\right| \leq C\left(\frac{\eta}{\left(\sigma^{\varepsilon}\right)^{2}}\right) \sup _{y \in I_{\sigma} \varepsilon}\left(\frac{|x-y|}{h^{2}}\right),
$$

because for all $y \in C_{k},\left(\theta^{\varepsilon, \eta}-\omega_{0}^{\eta}\right)(y)=\left(\theta^{\varepsilon, \eta}-\omega_{0}^{\eta}\right)\left(y_{k}\right)$ and

$$
\left|\exp \left(-\frac{(x-y)^{2}}{h}\right)-\exp \left(-\frac{\left(x-y_{k}\right)^{2}}{h}\right)\right| \leq C \eta \sup _{y \in I_{\sigma} \varepsilon}\left(\frac{|x-y|}{h}\right) .
$$

Then we write

$$
\int_{I_{\sigma \varepsilon}}(2 \pi h)^{-1}\left(\theta^{\varepsilon, \eta}-\omega_{0}^{\eta}\right)(y) \exp \left(-\frac{(x-y)^{2}}{h}\right) \mathrm{d} y=\int_{\mathbb{R}^{2}}(2 \pi h)^{-1}\left(\theta^{\varepsilon, \eta}-\omega_{0}^{\eta}\right)(y) \exp \left(-\frac{(x-y)^{2}}{h}\right) \mathrm{d} y
$$

and

$$
\begin{aligned}
\mid \int_{\mathbb{R}^{2}}(2 \pi h)^{-1}\left(\theta^{\varepsilon, \eta}-\omega_{0}^{\eta}\right)(y) \exp \left(-\frac{(x-y)^{2}}{h}\right) & -\left(\theta^{\varepsilon}-\omega_{0}\right)(x) \mid \mathrm{d} y \\
& \leq \int_{\mathbb{R}^{2}}(2 \pi h)^{-1}\left|\left(\theta^{\varepsilon, \eta}-\omega_{0}^{\eta}\right)(y)-\left(\theta^{\varepsilon}-\omega_{0}\right)(x)\right| \exp \left(-\frac{(x-y)^{2}}{h}\right) \mathrm{d} y .
\end{aligned}
$$

Lastly, we use the following estimate

$$
\begin{aligned}
\left|\left(\theta^{\varepsilon, \eta}-\omega_{0}^{\eta}\right)(y)-\left(\theta^{\varepsilon}-\omega_{0}\right)(x)\right| & \leq\left|\left(\theta^{\varepsilon, \eta}-\omega_{0}^{\eta}\right)(y)-\left(\theta^{\varepsilon}-\omega_{0}\right)(y)\right|+\left|\left(\theta^{\varepsilon}-\omega_{0}\right)(y)-\left(\theta^{\varepsilon}-\omega_{0}\right)(x)\right| \\
& \leq C(\eta+|x-y|) \sup \left(\left|\nabla\left(\theta^{\varepsilon}-\omega_{0}\right)\right|\right),
\end{aligned}
$$

to obtain for $|x| \leq \frac{1}{\sigma^{\varepsilon}}$

$$
|f(x)|=\left|\theta^{\varepsilon}(x)-\rho^{\varepsilon, h}(0, x)-\omega_{0}(x)\right| \leq C\left(\frac{\sqrt{h}}{\gamma^{\varepsilon}}+\frac{\eta}{\gamma^{\varepsilon}}+\frac{\eta}{\left(\sigma^{\varepsilon}\right)^{3} h^{2}}\right),
$$

and $|f(x)|=0$ if $|x|>\frac{1}{\sigma^{\varepsilon}}$.

We recall that $\nabla \theta^{\varepsilon} \sim \frac{1}{\gamma^{\varepsilon}}$ and that

$$
\int_{\mathbb{R}^{2}}|x-y| \exp \left(-\frac{(x-y)^{2}}{h}\right) \mathrm{d} y \leq C(\sqrt{h})^{3} .
$$


Then, we have

$$
\|f\|_{L^{2}}^{2}=\int|f(x)|^{2} \mathrm{~d} x \leq C \frac{1}{\left(\sigma^{\varepsilon}\right)^{2}}\left(\frac{\sqrt{h}}{\gamma^{\varepsilon}}+\frac{\eta}{\gamma^{\varepsilon}}+\frac{\eta}{\left(\sigma^{\varepsilon}\right)^{3} h^{2}}\right)^{2}
$$

and

$$
\||x| f\|_{L^{1}}=\int|x||f(x)| \mathrm{d} x \leq C \frac{1}{\left(\sigma^{\varepsilon}\right)^{3}}\left(\frac{\sqrt{h}}{\gamma^{\varepsilon}}+\frac{\eta}{\gamma^{\varepsilon}}+\frac{\eta}{\left(\sigma^{\varepsilon}\right)^{3} h^{2}}\right) .
$$

If we choose $\sigma^{\varepsilon}$ such that $\left(\frac{\sqrt{h}}{\gamma^{\varepsilon}}+\frac{\eta}{\gamma^{\varepsilon}}+\frac{\eta}{\left(\sigma^{\varepsilon}\right)^{3} h^{2}}\right)=\left(\sigma^{\varepsilon}\right)^{3} \mu(\varepsilon, h)$, with $\mu(\varepsilon, h) \stackrel{\varepsilon, h \rightarrow 0}{\longrightarrow} 0$, the estimate (3.5) implies that assumption (1.8) is satisfied.

\subsubsection{Condition on the kinetic energy}

Note

$$
I=\varepsilon \int \sum_{k \in I_{\eta}} \lambda_{k}\left|h \nabla \psi_{k}^{\varepsilon, h}(0, x)-i G^{\varepsilon}(x) \psi_{k}^{\varepsilon, h}(0, x)\right|^{2} \mathrm{~d} x
$$

with $G^{\varepsilon}(x)=\frac{{ }^{\perp} x}{2 \varepsilon}$.

The second assumption (1.9) will hold if $I$ goes to zero when $\varepsilon$ goes to zero. We have

$$
\begin{aligned}
I= & \varepsilon \int_{x} \sum_{k \in I_{\eta}} \lambda_{k}\left[h^{2}\left(i \frac{G^{\varepsilon}\left(y_{k}\right)}{h}-\frac{\left(x-y_{k}\right)}{h}\right) \psi_{k}^{\varepsilon, h}(0, x)\left(-i \frac{G^{\varepsilon}\left(y_{k}\right)}{h}-\frac{\left(x-y_{k}\right)}{h}\right) \bar{\psi}_{k}^{\varepsilon, h}(0, x)\right. \\
& \left.-2 G^{\varepsilon}(x) h \frac{G^{\varepsilon}\left(y_{k}\right)}{h} \psi_{k}^{\varepsilon, h}(0, x) \bar{\psi}_{k}^{\varepsilon, h}(0, x)+\left|G^{\varepsilon}(x)\right|^{2} \psi_{k}^{\varepsilon, h}(0, x) \bar{\psi}_{k}^{\varepsilon, h}(0, x)\right] \\
= & \varepsilon \int_{x} \sum_{k \in I_{\eta}} \lambda_{k}\left(\frac{h^{2}}{h^{2}}\left|G^{\varepsilon}\left(y_{k}\right)\right|^{2}+h^{2} \frac{\left(x-y_{k}\right)^{2}}{h^{2}}-2 \frac{h}{h} G^{\varepsilon}(x) G^{\varepsilon}\left(y_{k}\right)+\left|G^{\varepsilon}(x)\right|^{2}\right) \psi_{k}^{\varepsilon, h}(0, x) \bar{\psi}_{k}^{\varepsilon, h}(0, x),
\end{aligned}
$$

which becomes after simplification,

$$
I=\varepsilon \int_{x} \sum_{k \in I_{\eta}} \lambda_{k}\left(\left|G^{\varepsilon}\left(y_{k}\right)-G^{\varepsilon}(x)\right|^{2}+h^{2} \frac{\left(x-y_{k}\right)^{2}}{h^{2}}\right) \psi_{k}^{\varepsilon, h}(0, x) \bar{\psi}_{k}^{\varepsilon, h}(0, x) .
$$

Equality $G_{i}^{\varepsilon}\left(y_{k}\right)-G_{i}^{\varepsilon}(x)=\frac{{ }^{\perp} y_{k}-x}{\varepsilon}$ allows us to estimate $I$ and to obtain

$$
\begin{aligned}
I & \leq C \varepsilon \sum_{k \in I_{\eta}} \lambda_{k}\left(1+\frac{1}{\varepsilon^{2}}\right) \int_{x}\left(x-y_{k}\right)^{2}(2 \pi h)^{-\frac{d}{2}} \mathrm{e}^{-2 \frac{\left(x-y_{k}\right)^{2}}{h}} \mathrm{~d} x \\
& \leq C \sum_{k \in I_{\eta}} \lambda_{k} h\left(\varepsilon+\frac{1}{\varepsilon}\right) \sim \frac{h}{\left(\sigma^{\varepsilon}\right)^{2}}\left(\varepsilon+\frac{1}{\varepsilon}\right) \stackrel{\varepsilon, h \rightarrow 0}{\longrightarrow} 0,
\end{aligned}
$$

if $h=o\left(\left(\sigma^{\varepsilon}\right)^{2} \varepsilon\right)$.

In conclusion, let $\sigma^{\varepsilon}, \gamma^{\varepsilon}$ (that we will fix equal to $\varepsilon^{2}$ ) and $\mu(\varepsilon)$ be three functions of $\varepsilon$ which go to zero when $\varepsilon$ goes to zero, we choose $h$, such that $\sqrt{h}=\left(\sigma^{\varepsilon}\right)^{3} \gamma^{\varepsilon} \mu(\varepsilon)$ to have $h=o\left(\left(\sigma^{\varepsilon}\right)^{2} \varepsilon\right)$ and we choose $\eta$ such that $\eta=\mu(\varepsilon)\left(\sigma^{\varepsilon}\right)^{3} \inf \left(\gamma^{\varepsilon} ; h^{2}\left(\sigma^{\varepsilon}\right)^{3}\right)$. 


\section{Properties of the potential}

We give here the properties of the potential which are necessary for the proof of the conservation of energy.

Proposition 4.1. The parameter $\varepsilon$ being fixed, the potential $\phi^{\varepsilon}(t, \cdot)$ satisfies the following properties:

$$
\phi^{\varepsilon}(t, \cdot) \in L^{\infty}\left(\mathbb{R}^{2}\right), \quad \nabla \phi^{\varepsilon}(t, \cdot) \in L^{\infty}\left(\mathbb{R}^{2}\right) \cap L^{2}\left(\mathbb{R}^{2}\right) \text { and } \partial_{t} \nabla \phi^{\varepsilon}(t, \cdot) \in L^{2}\left(\mathbb{R}^{2}\right) .
$$

We recall that $\phi^{\varepsilon}, \theta^{\varepsilon}$ and $\rho^{\varepsilon}$ satisfy (1.3) and that $\int\left(\theta^{\varepsilon}-\rho^{\varepsilon}\right)=0$.

Proof. We use results from [1] stating that

$$
\begin{aligned}
\left|\phi^{\varepsilon}(x)\right| & \leq C\left(\left\|(|y|+1)\left(\theta^{\varepsilon}-\rho^{\varepsilon}\right)\right\|_{L_{y}^{1}\left(\mathbb{R}^{2}\right)}+\left\|(|y|+1)^{\frac{1}{2}}\left(\theta^{\varepsilon}-\rho^{\varepsilon}\right)\right\|_{L_{y}^{2}\left(\mathbb{R}^{2}\right)}\right), \\
\left|\nabla \phi^{\varepsilon}(x)\right| & \leq C\left(\left\|\theta^{\varepsilon}-\rho^{\varepsilon} \mid\right\|_{L^{3}\left(\mathbb{R}^{2}\right)}+\left\|\theta^{\varepsilon}-\rho^{\varepsilon}\right\|_{L^{1}\left(\mathbb{R}^{2}\right)}\right), \\
\left\|\nabla \phi^{\varepsilon}\right\|_{L^{2}\left(\mathbb{R}^{2}\right)} & \leq C\left(\left\||| \mid\left(\theta^{\varepsilon}-\rho^{\varepsilon}\right)\right\|_{L_{y}^{1}\left(\mathbb{R}^{2}\right)}+\left\|\theta^{\varepsilon}-\rho^{\varepsilon}\right\|_{L^{2}\left(\mathbb{R}^{2}\right)}\right) .
\end{aligned}
$$

Moreover, using the Sobolev injection $\left(W^{1, N}\left(\mathbb{R}^{N}\right) \subset L^{q}\left(\mathbb{R}^{N}\right)\right.$ for $q \in[N, \infty[)$, the authors of [1] show that

$$
\begin{aligned}
\left\|\rho^{\varepsilon}\right\|_{L^{p}\left(\mathbb{R}^{2}\right)} & \leq \sum \lambda_{m}\left\|\gamma_{m}\right\|_{L^{2 p}\left(\mathbb{R}^{2}\right)}^{2} \leq C|| \Gamma \|_{H^{1}\left(\mathbb{R}^{2}, \lambda\right)}^{2}, 1 \leq p<\infty \\
\left\|(1+|y|) \rho^{\varepsilon}\right\|_{L_{y}^{1}\left(\mathbb{R}^{2}\right)} & \leq\left. 2|| \Gamma\right|_{L^{2}\left(\mathbb{R}^{2}, \lambda\right)} ^{2}+\left\|\left|\|y \mid \Gamma\|_{L_{y}^{2}\left(\mathbb{R}^{2}, \lambda\right)}^{2}\right.\right. \\
\left\|(1+|y|)^{\frac{1}{2}} \rho^{\varepsilon}\right\|_{L_{y}^{2}\left(\mathbb{R}^{2}\right)} & \leq C\left(\|(1+|y|) \Gamma\|_{L_{y}^{2}\left(\mathbb{R}^{2}, \lambda\right)}\right)^{\frac{1}{2}}\left(\|\Gamma\|_{L^{6}\left(\mathbb{R}^{2}, \lambda\right)}\right)^{\frac{3}{2}} \\
& \leq C\left(\|(1+|y|) \Gamma\|_{L_{y}^{2}\left(\mathbb{R}^{2}, \lambda\right)}\right)^{\frac{1}{2}}\left(\|\Gamma\|_{H^{1}\left(\mathbb{R}^{2}, \lambda\right)}\right)^{\frac{3}{2}}
\end{aligned}
$$

This proves the first two assertions because $\Gamma \in H^{1}\left(\mathbb{R}^{2}, \lambda\right)$ and $|y| \Gamma \in L^{2}\left(\mathbb{R}^{2}, \lambda\right)$.

We also have

$$
\|\left.\nabla \partial_{t} \phi^{\varepsilon}\right|_{L^{2}\left(\mathbb{R}^{2}\right)} \leq C\left(\left\||y|\left(\partial_{t} \rho^{\varepsilon}\right)\right\|_{L_{y}^{1}\left(\mathbb{R}^{2}\right)}+\left\|\partial_{t} \rho^{\varepsilon}\right\|_{L^{2}\left(\mathbb{R}^{2}\right)}\right) .
$$

And equation $\partial_{t} \rho^{\varepsilon}=\sum \lambda_{m}\left(\partial_{t} \gamma_{m} \bar{\gamma}_{m}+\gamma_{m} \partial_{t} \bar{\gamma}_{m}\right)$ implies that

$$
\left\|| | y \left|( \partial _ { t } \rho ^ { \varepsilon } ) \left\|_{L_{y}^{1}\left(\mathbb{R}^{2}\right)} \leq C\left|\|y \mid \Gamma\|_{L_{y}^{2}\left(\mathbb{R}^{2}, \lambda\right)}\left\|\partial_{t} \Gamma\right\|_{L^{2}\left(\mathbb{R}^{2}, \lambda\right)}<C\right.\right.\right.\right.
$$

since the Schrödinger equation implies that $\partial_{t} \Gamma \in L^{2}\left(\mathbb{R}^{2}, \lambda\right)$.

Finally, we get

$$
\left\|\partial_{t} \rho^{\varepsilon}\right\|_{L^{2}\left(\mathbb{R}^{2}\right)}^{2} \leq C\left\|\partial_{t} \Gamma\right\|_{L^{2}\left(\mathbb{R}^{2}, \lambda\right)}\left\|\rho^{\varepsilon}\right\|_{L^{\infty}} .
$$

The right term is finite because $W^{m, p}\left(\mathbb{R}^{2}\right) \subset L^{\infty}\left(\mathbb{R}^{2}\right)$ if $\frac{1}{p}-\frac{m}{2}<0($ e.g. $m=2$ and $p=2)$ and then $\rho^{\varepsilon}$ belongs to $L^{\infty}\left(\mathbb{R}^{2}\right)$. Indeed $\Gamma \in L^{\infty}\left(\mathbb{R}^{2}, \lambda\right)$ because $\Gamma \in W^{2,2}\left(\mathbb{R}^{2}, \lambda\right)$.

\section{Proof of Proposition 2.4}

Let $\left(\psi_{k}^{\varepsilon}\right)_{k \in \mathbb{Z}^{2}} \in C([0, T] ; Y) \cap C^{1}([0, T] ; X)$ be a solution of (1.2) and (1.3),

$$
\left|h \nabla \psi_{k}^{\varepsilon}+i \frac{{ }^{\perp} x}{2 \varepsilon} \psi_{k}^{\varepsilon}\right|^{2}=\left|h \nabla \psi_{k}^{\varepsilon}\right|^{2}+2 J^{\varepsilon} \cdot \frac{{ }^{\perp} x}{2 \varepsilon}+\frac{1}{4} \frac{|x|^{2}}{\varepsilon} \rho,{ }^{\varepsilon}
$$


and then

$$
\frac{\mathrm{d}}{\mathrm{d} t} \tilde{H}^{\varepsilon}(t)=\sum_{k} \lambda_{k} \frac{\varepsilon}{2} \frac{\mathrm{d}}{\mathrm{d} t} \int h \nabla \psi_{k}^{\varepsilon} \cdot h \nabla \bar{\psi}_{k}^{\varepsilon}+\frac{1}{8} \int \frac{|x|^{2}}{\varepsilon} \partial_{t} \rho^{\varepsilon}+\frac{1}{2} \int{ }^{\perp} x \cdot \partial_{t} J^{\varepsilon}+\int \partial_{t} \nabla \phi^{\varepsilon} \cdot \nabla \phi^{\varepsilon}
$$

First of all, let us compute the first right-hand side term of (5.1) using the Schrödinger equation (1.2). Note that $\partial_{t} \nabla \psi^{\varepsilon} \in H^{-1}\left(\mathbb{R}^{2}, \lambda\right)$ because (1.2) ensures that $\partial_{t} \psi^{\varepsilon}(t, \cdot) \in L^{2}\left(\mathbb{R}^{2}, \lambda\right)$ since $\Delta \psi^{\varepsilon}(t, \cdot) \in L^{2}\left(\mathbb{R}^{2}, \lambda\right)$.

We define

$$
I=\frac{\mathrm{d}}{\mathrm{d} t} \sum_{k} \lambda_{k} \frac{\varepsilon}{2} \int h \nabla \psi_{k}^{\varepsilon} \cdot h \nabla \bar{\psi}_{k}^{\varepsilon}
$$

and we have

$$
\begin{aligned}
I= & \sum_{k} \lambda_{k} \frac{\varepsilon}{2}\left\langle h^{2} \partial_{t} \nabla \psi_{k}^{\varepsilon} ; \nabla \bar{\psi}_{k}^{\varepsilon}\right\rangle_{H^{-1}, H^{1}}+h^{2}\left\langle\partial_{t} \nabla \bar{\psi}_{k}^{\varepsilon} ; \nabla \psi_{k}^{\varepsilon}\right\rangle_{H^{-1}, H^{1}} \\
= & -\frac{i}{2} \varepsilon h\left[\sum_{k} \lambda_{k}\left\langle\nabla\left(-\frac{h^{2}}{2} \Delta \psi_{k}^{\varepsilon}-\frac{i h}{2 \varepsilon}\left({ }^{\perp} x \cdot \nabla\right) \psi_{k}^{\varepsilon}+\frac{|x|^{2}}{8 \varepsilon^{2}} \psi_{k}+\frac{1}{\varepsilon} \phi^{\varepsilon} \psi_{k}^{\varepsilon}\right) ; \nabla \bar{\psi}_{k}\right\rangle_{H^{-1}, H^{1}}\right. \\
& \left.+\sum_{k} \lambda_{k}\left\langle\nabla \psi_{k} ; \nabla\left(\frac{h^{2}}{2} \Delta \bar{\psi}_{k}^{\varepsilon}-\frac{i h}{2 \varepsilon}\left({ }^{\perp} x \cdot \nabla\right) \bar{\psi}_{k}^{\varepsilon}-\frac{|x|^{2}}{8 \varepsilon^{2}} \bar{\psi}_{k}-\frac{1}{\varepsilon} \phi^{\varepsilon} \bar{\psi}_{k}^{\varepsilon}\right)\right\rangle_{H^{1}, H^{-1}}\right]
\end{aligned}
$$

and then, it follows that

$$
\begin{aligned}
I= & \sum_{k} \lambda_{k} \int \frac{h \varepsilon}{2 i} \frac{h^{2}}{2}\left(\Delta \psi_{k}^{\varepsilon} \Delta \bar{\psi}_{k}^{\varepsilon}-\Delta \psi_{k}^{\varepsilon} \Delta \bar{\psi}_{k}^{\varepsilon}\right)+\sum_{k} \lambda_{k} \int \frac{h}{2 i} \nabla\left(\frac{|x|^{2}}{8 \varepsilon}\right) \cdot\left(\nabla \bar{\psi}_{k}^{\varepsilon} \psi_{k}^{\varepsilon}-\nabla \psi_{k}^{\varepsilon} \bar{\psi}_{k}^{\varepsilon}\right) \\
& +\sum_{k} \lambda_{k} \int \nabla\left(\phi^{\varepsilon}\right) \cdot\left(\nabla \bar{\psi}_{k}^{\varepsilon} \psi_{k}^{\varepsilon}-\nabla \psi_{k}^{\varepsilon} \bar{\psi}_{k}^{\varepsilon}\right) \frac{h}{2 i}+\sum_{k} \lambda_{k} \frac{h^{2}}{4}\left\langle\left(\nabla\left({ }^{\perp} x \cdot \nabla \psi_{k}^{\varepsilon}\right) ; \nabla \bar{\psi}_{k}^{\varepsilon}\right\rangle_{H^{1}, H^{-1}}\right. \\
& \left.+\sum_{k} \lambda_{k} \frac{h^{2}}{4}\left\langle\nabla\left({ }^{\perp} x \cdot \nabla \bar{\psi}_{k}^{\varepsilon}\right) ; \nabla \psi_{k}^{\varepsilon}\right)\right\rangle_{H^{-1}, H^{1}} \\
= & \int\left(\frac{|x|^{2}}{8 \varepsilon}+\phi^{\varepsilon}\right) \operatorname{div} J^{\varepsilon}-\frac{h^{2}}{4} \sum_{k} \lambda_{k} \int \operatorname{div}\left({ }^{\perp} x\left(\nabla \psi_{k}^{\varepsilon} \cdot \nabla \bar{\psi}_{k}^{\varepsilon}\right)\right) \\
= & \int\left(\frac{|x|^{2}}{8 \varepsilon}+\phi^{\varepsilon}\right) \operatorname{div} J^{\varepsilon} .
\end{aligned}
$$


Each of the above integrals are well defined. Indeed, we have

$$
\begin{aligned}
& \left|\sum_{k} \lambda_{k} \int \frac{h}{2 i} \nabla\left(\frac{|x|^{2}}{8 \varepsilon}\right) \cdot\left(\nabla \bar{\psi}_{k}^{\varepsilon} \psi_{k}^{\varepsilon}-\nabla \psi_{k}^{\varepsilon} \bar{\psi}_{k}^{\varepsilon}\right)\right| \leq C \sum_{k} \lambda_{k}\left\|x \cdot \nabla \psi_{k}\right\|_{L^{2}}\left\|\psi_{k}\right\|_{L^{2}} \\
& \leq C\left(\sum_{k} \lambda_{k}\left\|x \cdot \nabla \psi_{k}\right\|_{L^{2}}^{2}\right)^{\frac{1}{2}}\left(\sum_{k} \lambda_{k}\left\|\psi_{k}\right\|_{L^{2}}^{2}\right)^{\frac{1}{2}} \\
& =C\|x \cdot \nabla \psi\|_{L^{2}(\lambda)}\|\psi\|_{L^{2}(\lambda)}, \\
& \left|\int \frac{|x|^{2}}{8 \varepsilon} \operatorname{div} J^{\varepsilon}\right| \leq C\left(|||x|^{2} \psi\left\|_{L^{2}(\lambda)}|| \Delta \psi\right\|_{L^{2}(\lambda)}+\left|\|x \mid \cdot \nabla \psi\|_{L^{2}(\lambda)}^{2}\right)\right. \\
& \left|\sum_{k} \lambda_{k} \int \operatorname{div}\left({ }^{\perp} x\left(\nabla \psi_{k}^{\varepsilon} \cdot \nabla \bar{\psi}_{k}^{\varepsilon}\right)\right)\right| \leq|||x| \nabla \psi^{\varepsilon}\left\|_{L^{2}(\lambda)}|| \psi^{\varepsilon}\right\|_{H^{2}(\lambda)}, \\
& \left\|\operatorname{div} J^{\varepsilon}\right\|_{L^{1}} \leq\left\|\psi^{\varepsilon}\right\|_{L^{2}(\lambda)}\left\|\Delta \psi^{\varepsilon}\right\|_{L^{2}(\lambda)}+\left\|\nabla \psi^{\varepsilon}\right\|_{L^{2}(\lambda)}^{2},
\end{aligned}
$$

and $\phi^{\varepsilon}(t, \cdot) \in L^{\infty}\left(\mathbb{R}^{2}\right), \nabla \phi^{\varepsilon}(t, \cdot) \in L^{\infty}\left(\mathbb{R}^{2}, \mathbb{R}^{2}\right)$ (see Sect. 4). Moreover, the integration by parts can be performed because ${ }^{\perp} x\left(\nabla \psi_{k}^{\varepsilon} \cdot \nabla \bar{\psi}_{k}^{\varepsilon}\right)$ belongs to $L^{1}\left(\mathbb{R}^{2}\right)$. We recall that $\psi^{\varepsilon} \in Y$ and then for a fixed $\varepsilon$, the right terms are finite.

In a second step, we now consider the third right-hand side term in (5.1). We rewrite it under the following equivalent form using $(2.5)$

$$
\begin{aligned}
\frac{1}{2} \int{ }^{\perp} x \cdot \partial_{t} J^{\varepsilon}= & -\sum_{k} \lambda_{k} \frac{1}{2} \operatorname{Re} \int{ }^{\perp} x \cdot \nabla:\left(h \nabla \psi_{k}^{\varepsilon} \otimes h \nabla \bar{\psi}_{k}^{\varepsilon}\right)-\frac{1}{2 \varepsilon} \int{ }^{\perp} x \cdot \nabla \phi^{\varepsilon} \rho^{\varepsilon} \\
& +\frac{1}{2} \int{ }^{\perp} x \cdot \frac{1}{2 \varepsilon}{ }^{\perp} J^{\varepsilon}-\frac{1}{2} \int \frac{1}{2 \varepsilon}{ }^{\perp} x \cdot\left({ }^{\perp} x \cdot \nabla\right) J^{\varepsilon}-\frac{1}{2} \int{ }^{\perp} x \cdot \nabla \frac{|x|^{2}}{8 \varepsilon^{2}} \rho^{\varepsilon},
\end{aligned}
$$

since ${ }^{\perp} x \cdot \nabla:\left(h \nabla \psi_{k}^{\varepsilon} \otimes h \nabla \bar{\psi}_{k}^{\varepsilon}\right)={ }^{\perp} x_{i} \partial_{j}\left(h^{2} \partial_{i} \psi_{k}^{\varepsilon} \partial_{j} \bar{\psi}_{k}^{\varepsilon}\right)$.

The previous integrals are well defined thanks to the following upper estimates

$$
\begin{aligned}
& \left|\sum_{k} \lambda_{k} \iint{ }^{\perp} x \cdot \nabla:\left(h \nabla \psi_{k}^{\varepsilon} \otimes h \nabla \bar{\psi}_{k}^{\varepsilon}\right)\right| \leq\left\|\psi^{\varepsilon}||_{H^{2}(\lambda)}|||x| \cdot \nabla \psi\right\|_{L^{2}(\lambda)}, \\
& \left|\int{ }^{\perp} x . \nabla \phi^{\varepsilon} \rho^{\varepsilon}\right| \leq\left\|\nabla \phi^{\varepsilon}\right\|_{L^{\infty}}|||x| \psi^{\varepsilon}\left\|_{L^{2}(\lambda)}|| \psi^{\varepsilon}\right\|_{L^{2}(\lambda)}, \\
& \left|\int{ }^{\perp} x \cdot J\right| \leq\left\|{ }^{\perp} x \cdot \nabla \psi^{\varepsilon}\right\|_{L^{2}(\lambda)}\left\|\psi^{\varepsilon}\right\|_{L^{2}(\lambda)}, \\
& \left|\int{ }^{\perp} x \cdot\left({ }^{\perp} x . \nabla\right) J^{\varepsilon}\right| \leq\left\|| | x | ^ { 2 } \psi ^ { \varepsilon } \left|\left\|_{L^{2}(\lambda)}|| \psi^{\varepsilon}\right\|_{H^{2}(\lambda)}+\left\|{ }^{\perp} x \cdot \nabla \psi^{\varepsilon}\right\|_{L^{2}(\lambda)}^{2} .\right.\right.
\end{aligned}
$$


After simplification, we have

$$
\begin{aligned}
\frac{1}{2} \int{ }^{\perp} x \cdot \partial_{t} J^{\varepsilon}= & \sum_{k} \lambda_{k} \operatorname{Re} \int h^{2}\left(\partial_{2} \psi_{k}^{\varepsilon} \partial_{1} \bar{\psi}_{k}^{\varepsilon}-\partial_{2} \bar{\psi}_{k}^{\varepsilon} \partial_{1} \psi_{k}^{\varepsilon}\right) \\
& +\frac{1}{4 \varepsilon} \int x \cdot J^{\varepsilon}+\frac{1}{4 \varepsilon} \int D\left({ }^{\perp} x\right):\left(J^{\varepsilon} \otimes{ }^{\perp} x\right)-\int{ }^{\perp} x \cdot \frac{x}{8 \varepsilon^{2}} \rho^{\varepsilon}+\frac{1}{2 \varepsilon} \int{ }^{\perp} x \cdot \nabla \rho^{\varepsilon} \phi^{\varepsilon} \\
= & \frac{1}{4 \varepsilon} \int x \cdot J^{\varepsilon}-\frac{1}{4 \varepsilon} \int x \cdot J^{\varepsilon}+\frac{1}{2 \varepsilon} \int{ }^{\perp} x \cdot \nabla \rho^{\varepsilon} \phi^{\varepsilon} \\
= & \frac{1}{2 \varepsilon} \int{ }^{\perp} x \cdot \nabla \rho^{\varepsilon} \phi^{\varepsilon}=\int \phi^{\varepsilon} \operatorname{div}\left(\frac{{ }^{\perp} x}{2 \varepsilon} \rho^{\varepsilon}\right) .
\end{aligned}
$$

The last term is finite because

$$
\left|\int{ }^{\perp_{x}} x . \nabla \rho^{\varepsilon} \phi^{\varepsilon}\right| \leq\left\|\phi^{\varepsilon}\right\|_{L^{\infty}}\left\|^{\perp} x \cdot \nabla \psi^{\varepsilon}\right\|_{L^{2}(\lambda)}\left\|\psi^{\varepsilon}\right\|_{L^{2}(\lambda)} .
$$

Finally, we obtain

$$
\begin{aligned}
\frac{\mathrm{d}}{\mathrm{d} t} \tilde{H}^{\varepsilon}(t) & =\int\left(\frac{|x|^{2}}{8 \varepsilon}+\phi^{\varepsilon}\right) \operatorname{div} J^{\varepsilon}+\frac{1}{8} \int \frac{|x|^{2}}{\varepsilon} \partial_{t} \rho^{\varepsilon}+\int \phi^{\varepsilon} \operatorname{div}\left(\frac{{ }^{\perp} x}{2 \varepsilon} \rho^{\varepsilon}\right)+\int \partial_{t} \nabla \phi^{\varepsilon} \cdot \nabla \phi^{\varepsilon} \\
& =\int \phi^{\varepsilon} \operatorname{div} \tilde{J}^{\varepsilon}-\int \partial_{t} \Delta \phi^{\varepsilon} \phi^{\varepsilon} \\
& =-\int \phi^{\varepsilon} \partial_{t} \rho^{\varepsilon}-\int \partial_{t} \Delta \phi^{\varepsilon} \phi^{\varepsilon}=0 .
\end{aligned}
$$

We have obtained that the energy $\tilde{H}^{\varepsilon}$ defined by (2.6) does not depend on time and this concludes the proof of Proposition 2.4.

To justify the sense of each integral of the previous computation, we use again some results of Section 4 , which are $\nabla \phi^{\varepsilon}(t, \cdot) \in L^{2}\left(\mathbb{R}^{2}, \mathbb{R}^{2}\right), \partial_{t} \nabla \phi^{\varepsilon}(t, \cdot) \in L^{2}\left(\mathbb{R}^{2}, \mathbb{R}^{2}\right)$ and the following results

$$
\left|\int \frac{|x|^{2}}{\varepsilon} \partial_{t} \rho^{\varepsilon}\right| \leq\left\|\left.|| x\right|^{2} \psi\right\|_{L^{2}(\lambda)}\left\|\partial_{t} \psi\right\|_{L^{2}(\lambda)}
$$

and

$$
\left|\int \phi^{\varepsilon} \operatorname{div} \tilde{J}^{\varepsilon}\right|=\left|\int \phi^{\varepsilon} \partial_{t} \rho^{\varepsilon}\right| \leq\left\|\phi^{\varepsilon}\right\|_{L^{\infty}}\left\|\partial_{t} \psi\right\|_{L^{2}(\lambda)}\|\psi\|_{L^{2}(\lambda)} .
$$

Acknowledgements. The author thanks Yann Brenier for helpful discussions and the European TMR network "Asymptotic Methods in Kinetic Theory" for its financial support.

\section{REFERENCES}

[1] A. Arnold and F. Nier, The two-dimensional Wigner-Poisson problem for an electron gas in the charge neutral case. Math. Methods Appl. Sci. 14 (1991) 595-613.

[2] Y. Brenier, Convergence of the Vlasov-Poisson system to the incompressible Euler equations. Comm. Partial Differential Equations 25 (2000) 737-754. 
[3] T. Cazenave, An introduction to nonlinear Schrödinger equations, in: Textos de méthodos Mathemàticas 26. Universidad Federal do Rio de Janeiro (1993).

[4] C. Cohen-Tannoudji, B. Diu and F. Laloë, Mécanique quantique. Hermann (1973).

[5] P. Gérard, P.A. Markowich, N.J. Mauser and F. Poupaud, Homogenization limits and Wigner transforms. Comm. Pure Appl. Math. 50 (1997) 323-379.

[6] J.-L. Lions, Quelques méthodes de résolution des problèmes aux limites non linéaires. Dunod, Gauthiers-Villars, Paris (1969).

[7] P.-L. Lions, Mathematical topics in fluid mechanics, Vol. 1. Incompressible models. Oxford Lecture in Mathematics and its Applications. Oxford University Press, New York (1996).

[8] P.-L. Lions and T. Paul, Sur les mesures de Wigner. Rev. Mat. Iberoamericana 9 (1993) 553-618.

[9] P.A. Markowich and N.J. Mauser, The classical limit of a self-consistent quantum-Vlasov equation in 3D. Math. Models Methods Appl. Sci. 3 (1993) 109-124.

[10] M. Puel, Convergence of the Schrödinger-Poisson system to the incompressible Euler equations. Preprint LAN, Université Paris VI (2001).

[11] M. Puel, Études variationnelle et asymptotique de problèmes en mécanique des fluides et des plasmas. Ph.D. thesis, Université Paris VI (2001).

To access this journal online:

www.edpsciences.org 